\title{
A simple model for molecular hydrogen chemistry coupled to radiation hydrodynamics
}

\author{
Sarah Nickerson, ${ }^{1 \star}$ Romain Teyssier, ${ }^{1}$ Joakim Rosdahl ${ }^{2,3}$ \\ ${ }^{1}$ Institute for Computational Science, University of Zürich, Winterthurerstrasse 190, CH-8057 Zürich, Switzerland \\ ${ }^{2}$ Leiden Observatory, Leiden University, PO Box 9513, NL-2300 RA, Leiden, the Netherlands \\ ${ }^{3}$ Centre for Astronomy Research, University of Lyon, 9 avenue Charles André, 69230 Saint-Genis-Laval, France
}

Accepted XXX. Received YYY; in original form ZZZ

\begin{abstract}
We introduce non-equilibrium molecular hydrogen chemistry into the radiationhydrodynamics code RAMSES-RT. This is an adaptive mesh refinement grid code with radiation hydrodynamics that couples the thermal chemistry of hydrogen and helium to moment-based radiative transfer with the Eddington tensor closure model. The $\mathrm{H}_{2}$ physics that we include are formation on dust grains, gas phase formation, collisional destruction, photodissociation, photoionisation, and self-shielding. In particular, we implement the first model for $\mathrm{H}_{2}$ self-shielding that is tied locally to moment-based radiative transfer by enhancing photo-destruction. This self-shielding from LymanWerner line overlap is critical to $\mathrm{H}_{2}$ formation and gas cooling. We can now track the non-equilibrium evolution of molecular, atomic, and ionised hydrogen species with their corresponding dissociating and ionising photon groups. Over a series of tests we show that our model works well compared to specialised photodissociation region codes. We successfully reproduce the transition depth between molecular and atomic hydrogen, molecular cooling of the gas, and a realistic Strömgren sphere embedded in a molecular medium. In this paper we focus on test cases to demonstrate the validity of our model on small scales, but our ultimate application in the future will be large-scale galactic simulations.
\end{abstract}

Key words: methods: numerical - molecular processes - radiative transfer

\section{INTRODUCTION}

The study of $\mathrm{H}_{2}$ in galaxies touches on an immense range of scales. Observations on the galactic, kpc scale show that $\mathrm{H}_{2}$ correlates with star formation (Wong \& Blitz 2002 Schruba et al. 2011 Leroy et al. 2013). On the pc scale are the molecular clouds themselves. Within the Milky Way their mass distribution follows a power law similar to that of the luminosity distribution of OB stars (Williams \& McKee 1997), and their velocity dispersion follows a power law that increases with radius (Larson 1981). These intermediate scale mechanics are influenced by, and in turn influence, both the galaxy-wide dynamics and the molecular level chemistry.

The typical giant molecular cloud (GMC) has an outer layer of atomic hydrogen (H I), which shields an inner core of $\mathrm{H}_{2}$ from interstellar radiation, and a $\mathrm{CO}$ core. GMCs have long been established to be the sites of star formation (McKee \& Ostriker 2007). Schmidt (1959) and Kennicutt (1998) demonstrate that the neutral hydrogen surface density corre-

^ E-mail: snickers@physik.uzh.ch lates to surface density of star formation (the K-S relation), while recent observations show that the $\mathrm{H}_{2}$ surface density correlates even more tightly to the star formation (e.g. Bigiel et al. 2008).

It is unclear whether there is a causation behind this correlation. What is known, however, is that $\mathrm{H}_{2}$ is an important coolant for interstellar gas (Gnedin \& Kravtsov 2011). Unfortunately, $\mathrm{H}_{2}$ is exceptionally difficult to directly observe owing to its lack of a dipole moment. This leads to GMC identification by CO content (Solomon et al. 1987). The $\mathrm{H}_{2}$ content can then be inferred by a conversion factor between the $\mathrm{CO}$ intensity and the column density of $\mathrm{H}_{2}$. This conversion factor has been extensively measured to be constant for the Milky Way, but further studies beyond the Milky Way show that it might depend on galaxy morphology and metallicity (Bolatto et al. 2013).

Smaller still than GMCs is the scale of the particles themselves and the chemistry by which hydrogen becomes molecular. Grains of interstellar dust serve as catalysts by which $\mathrm{HI}$ sticks and coalesces into $\mathrm{H}_{2}$ (Gould \& Salpeter 1963). $\mathrm{H}_{2}$ may also form by gas phase interactions, but this 
process is much slower and was only important in the early Universe when metals were scarce (Galli \& Palla 1998). At high temperatures $(T \gtrsim 1000 \mathrm{~K})$, collisions between $\mathrm{H}_{2}$ and other particles dissociate $\mathrm{H}_{2}$ into $\mathrm{H}$ I (Glover \& Abel 2008). $\mathrm{H}_{2}$ is also ionised by high energy photons $(h v \geq 15.42 \mathrm{eV}$, where $v$ is the photon frequency) (Abel et al. 1997). Finally, photons that fall into the Lyman-Werner (LW) band, 11.2 to $13.6 \mathrm{eV}$, dissociate $\mathrm{H}_{2}$. Outer regions of the GMCs absorb the LW photons at stronger wavelengths first, but allow for weaker wavelengths to penetrate further until they too are absorbed, and an outer $\mathrm{H}$ I layer forms to protect the inner $\mathrm{H}_{2}$ from LW photons.

In addition to the outer Hi layer in clouds, two processes shield $\mathrm{H}_{2}$ from radiation. The first is shielding by dust, and the second is $\mathrm{H}_{2}$ self-shielding. Only about $10 \%$ of LW absorption leads to $\mathrm{H}_{2}$ dissociation (Stecher \& Williams 1967) and the rest of the photons are destroyed without contributing to photodissociation (Sternberg et al. 2014). The absorption rate is highly dependent on the wavelength of the LW band (Abgrall et al. 1992 Haiman et al. 2000). Certain bands become optically thick at high $\mathrm{H}_{2}$ column densities, and dissociation is quashed, while bands with weaker absorption can still penetrate the cloud. Here an increase in the natural line width, due to Heisenberg uncertainty, leads to interference between the LW bands. Self-shielding is weakest at low column densities and increases further into the cloud. Hence, $\mathrm{H}_{2}$ self-shielding functions calculated from experiments are given in terms of the column density of $\mathrm{H}_{2}$, the most widely-used of which is from Draine \& Bertoldi (1996). Gnedin \& Draine (2014) update this function to account for turbulence in molecular clouds.

It is this range of scales, from the quantum mechanical nature of $\mathrm{H}_{2}$ self-shielding, to the far-reaching gravitational influence of a galaxy on GMCs, that makes simulating molecular chemistry challenging. It is modelled on the smallest scale in photodissociation region (PDR) codes and on the largest scale in galaxy codes.

PDRs are predominately neutral regions of the interstellar medium (ISM) in which far ultraviolet (UV) photons (6 $\mathrm{eV}<h v<13.6 \mathrm{eV})$ control the temperature and chemistry. They contain all of the atomic and at least $90 \%$ of molecular gas in the Milky Way, and are a major non-stellar source of infrared (IR) radiation in the ISM (Hollenbach \& Tielens 1999). PDR models are diverse, exhibiting different geometries, from one-dimensional to spherical, and are developed to study a range of phenomena. Many focus on interstellar clouds, both the clumps inside the clouds themselves and the boundaries between molecular clouds and ionised regions. Others study plasmas, circumstellar discs, planetary nebulae, the centre of the Milky Way, and the ratio between CO and $\mathrm{H}_{2}$. PDR models involve sophisticated chemical networks with species of hydrogen, carbon, oxygen, and silicone; detailed treatment of dust; radiative transfer of multiple photon groups; and the heating and cooling processes a cloud undergoes as a result of the interactions between the gas, dust, and photons. Roellig et al. (2007) is a first of its kind comparison study of ten PDR codes, consisting of a series of benchmark tests to highlight where the codes converge and understand where they differ.

The past decade has seen an array of methods to model the $\mathrm{H}_{2}$ chemistry in both semi-analytical galaxy models and hydrodynamical galaxy simulations. They explore the na- ture of the relationship between star formation and $\mathrm{H}_{2}$, test star formation recipes, and see how $\mathrm{H}_{2}$ affects the gas composition of galaxies. The semi-analytical models use equilibrium equations to find the $\mathrm{H}_{2}$ fraction (Fu et al. 2010 . Somerville et al. 2015, Xie et al. 2017) while hydrodynamic simulations use either equilibrium equations (Pelupessy et al. 2006, Robertson \& Kravtsov 2008 |Kuhlen et al. 2012 Halle \& Combes 2013; Hopkins et al. 2014; Thompson et al. 2014) or a series of non-equilibrium chemical networks (Gnedin et al. 2009, Gnedin \& Kravtsov 2011, Christensen et al. 2012, Tomassetti et al. 2014| Richings \& Schaye 2016 Hu et al. 2016, Capelo et al. 2017, Lupi et al.|2017, Pallottini et al. 2017, Katz et al. 2017). Equilibrium calculations have the advantage of speed but run on the assumption that the chemical species are in equilibrium with their environment. Non-equilibrium codes instead use local rates of destruction and creation of chemical species, and networks of rate equations.

To date, only three of these codes use radiative transfer with a non-equilibrium chemical network. Gnedin \& Kravtsov (2011) implement an $\mathrm{H}_{2}$-based star formation recipe for cosmological galaxy simulations and demonstrate that the molecular content of a galaxy and its K-S relation are sensitive to both the dust to gas ratio and the UV flux. In contrast Lupi et al. (2017)'s star formation recipe is independent of $\mathrm{H}_{2}$ content and still reproduces the K-S relation. Katz et al. (2017)'s method is the most similar to ours, modifying RAMSES-RT to track $\mathrm{H}_{2}$ in cosmological simulations for comparison to ALMA observations. Our $\mathrm{H}_{2}$ model differs from all of these in the $\mathrm{H}_{2}$ self-shielding approximation.

The models for $\mathrm{H}_{2}$ self-shielding, as mentioned above, describe the shielding as a function of cloud column density. Simulations, however, use the volume density and conversion is necessary. The most computationally simple way is to convert volume density into a column density using a Jeans length, a Sobolev length, or a Sobolev-like length. Non-local methods use neighbouring cells to compute a column density, but are more expensive (Wolcott-Green et al. (2011) provide an overview). Gnedin \& Kravtsov (2011) treat their conversion length as a free parameter to be computed, while Lupi et al. (2017) use a Jeans length, and Katz et al. (2017) use the cell width. These self-shielding functions then decrease the $\mathrm{H}_{2}$ destruction. We instead take full advantage of our radiative transfer to model self-shielding by enhancing photo-destruction in the LW band. In this way we do not need to use a volume-to-column density approximation.

In this work, we present the first model of $\mathrm{H}_{2}$ physics tied directly to moment-based radiative transfer by a local self-shielding approximation for the LW photo-destruction. RAMSES-RT is optimised for radiation-hydrodynamical galaxy simulations and photoionisation, but our new method also holds up under the conditions of PDR codes, thus linking the two regimes. Both radiative transfer and nonequilibrium calculations of $\mathrm{H}_{2}$ are important to study the problem of how $\mathrm{H}_{2}$ affects galaxies. We will be able to use this methodology for not only isolated disc galaxies, but also galaxies in a cosmological context. The combination of moment-based RT and the few photon groups required uniquely situates us to simulate $\mathrm{H}_{2}$ chemistry in cosmology.

RAMSES (Teyssier 2002) is an adaptive mesh refinement (AMR) code for N-body hydrodynamical galaxy simulations, both cosmological and isolated discs. RAMSES-RT 
(Rosdahl et al. 2013) implements radiation hydrodynamics for RAMSES, coupling photons to the non-equilibrium chemistry of the neutral and ionised species of hydrogen and helium. It utilises a moment-based method of radiative transfer, which unlike ray tracing is independent of the number of sources. This makes it ideal for galaxy simulations that host large numbers of stars. In this paper, the first of two, we present an upgrade to implement $\mathrm{H}_{2}$ chemistry into the RAMSES-RT code. Our tests show our $\mathrm{H}_{2}$ model's ability to match PDR code benchmarks and simulate realistic molecular Strömgren spheres. In a follow-up paper we will demonstrate the effects of our $\mathrm{H}_{2}$ model in galaxy simulations.

In Section 2 we give an overview of RAMSES-RT and our new implementation for $\mathrm{H}_{2}$ physics. In Section 3 , idealised tests prove the rigour of our method in zero, one, two, and three dimensions. We include comparisons to PDR codes and a Strömgren sphere embedded in a molecular medium. Finally, in Section 4, we summarise our findings and provide future directions for our current work.

\section{METHOD}

We begin with an overview of RAMSES and its radiative transfer features before diving into the specific details of the $\mathrm{H}_{2}$ physics. Previously, Ramses-RT only tracked $\mathrm{HI}$, H II, $\mathrm{He}$, He II, and He III. In the review we show where $\mathrm{H}_{2}$ is also included in the equations in order to provide a complete and updated picture.

\subsection{Overview of Ramses-RT}

RAMSES is an adaptive mesh refinement (AMR) hydrodynamical code with an N-body solver for stellar populations and dark matter, and a tree-based data structure grid for the gravitational potential and advection of gas (Teyssier 2002). The radiative transfer (RT) extension (Rosdahl et al. 2013) introduces radiative transfer coupled to the hydrodynamics in RAMSES, directly tracking photon groups that are tied to the non-equilibrium chemistry of H I, H II, He I, He II, and He III via photoionisation and heating. In this paper we introduce the non-equilibrium chemistry of $\mathrm{H}_{2}$ and include its index in this overview section. The full details of the $\mathrm{H}_{2}$ chemistry are in Section 2.3

In RAMSES-RT, radiation frequency is discretised into groups whose attributes are averaged over frequency ranges. Each gas cell at a given time is described by a state $\mathcal{U}=\left(\rho, \rho \mathbf{u}, E, \rho x_{\mathrm{H}}, \rho x_{\mathrm{H} I \mathrm{I}}, \rho x_{\mathrm{He} \mathrm{II}}, \rho x_{\mathrm{He} \mathrm{III}}, N_{i}, \mathbf{F}_{i}\right)$ (mass density, momentum density, energy density, H I fraction abundance, HII fraction abundance, He I fraction abundance, He II fraction abundance, photon density for each radiation group, and flux for each group). $\mathrm{H}_{2}$ and $\mathrm{He}$ I fractions are not tracked, but can be recovered from the other species fractions.

RAMSES-RT uses a moment-based approach by treating the photons as a sort of fluid, which renders the computational cost independent of the number of radiation sources. For galaxy simulations filled with stars, this makes momentbased much faster compared to the alternative of ray tracing. The main disadvantage of the moment-based method is that we need an approximate closure model for the pressure tensor (Equation 3). An exact treatment requires ray-tracing, which is computationally expensive, and we opt instead for a local method. One further hurdle is that in RAMSES-RT the radiative transfer is advanced explicitly in time, and in the free-streaming limit this leads to much smaller time steps for the RT as compared to pure hydrodynamic simulations. RAMSES-RT solves this problem with the reduced speed of light approach (Gnedin \& Abel 2001), which is a valid approximation as long as the light crossing time is shorter than the sound crossing, recombination, and advection time scales.

RAMSES-RT implements recombination emission from every gas cell, and it also provides the option of an on-thespot-approximation (OTSA) where recombination photons are assumed to be absorbed in the same gas cell thereby ignoring direct-to-ground-state recombinations. A later extension (Rosdahl \& Teyssier 2015) adds radiation pressure and dust absorption to RAMSES-RT.

\subsection{Moment-based radiative transfer}

We summarize here the moment-based approach in RAMSES$\mathrm{RT}$ as described in Rosdahl et al. (2013), with the addition of molecular hydrogen. Further sources Mihalas \& Mihalas (1984) and Aubert \& Teyssier (2008) outline this process in more detail.

$I_{v}(\mathbf{x}, \mathbf{n}, t)$ is the specific radiation intensity at a wavelength $v$, location $\mathbf{x}$, direction $\mathbf{n}$, and time $t$ in units erg $\mathrm{cm}^{-2} \mathrm{~s}^{-1} \mathrm{~Hz}^{-1} \mathrm{rad}^{-2}$. The evolution of the specific intensity is described by the equation of radiative transfer:

$\frac{1}{c_{r}} \frac{\partial I_{v}}{\partial t}+\mathbf{n} \cdot \nabla I_{v}=-\kappa_{v} I_{v}+\eta_{v}$

where $c_{r}$ is the reduced speed of light, $\kappa_{v}(\mathbf{x}, \mathbf{n}, t)$ is the gas opacity, and $\eta_{v}(\mathbf{x}, \mathbf{n}, t)$ is the source function. The time evolution of the photon number density $N_{v}$, and flux $\mathbf{F}_{v}$ are then extracted from Equation 1 by taking the zeroth and first angular moments:

$$
\begin{aligned}
\frac{\partial N_{v}}{\partial t}+\nabla \cdot \mathbf{F}_{v} & =-\sum_{j}^{\mathrm{H}_{2}, \mathrm{H} \mathrm{I}, \mathrm{He} \mathrm{I}, \mathrm{He} \mathrm{II}} n_{j} \sigma_{v j} c_{r} N_{v} \\
& -\kappa_{P d} \rho_{d} c_{r} N_{\nu}+\dot{N}_{v}^{\star}+\dot{N}_{v}^{r e c}, \\
\frac{\partial \mathbf{F}_{v}}{\partial t}+c_{r}^{2} \nabla \cdot \mathbb{P}_{v} & =-\sum_{j}^{\mathrm{H}_{2}, \mathrm{H} \mathrm{I}, \mathrm{He} \text { I,He II }} n_{j} \sigma_{v j} c_{r} \mathbf{F}_{v} \\
& -\kappa_{R d} \rho_{d} c_{r} \mathbf{F}_{\nu},
\end{aligned}
$$

where $n_{j}$ is the number density of species $j, \sigma_{v j}$ is the ionisation/dissociation cross-section between photons with frequency $v$ and species $j, \kappa_{P d}$ and $\kappa_{R d}$ are the Planck and Rosseland dust opacities, $\rho_{d}$ is the dust volume density, $\dot{N}_{v}^{\star}$ is the number of photons injected by stars, $\dot{N}_{v}^{r e c}$ is the number of photons injected by gas recombination when OTSA is off, and $\mathbb{P}_{v}$ is the radiative pressure tensor. The dust density is given by $\rho_{d} \equiv Z f_{d} \rho$, where $Z$ is the metallicity, $f_{d}$ is the fraction of gas that holds dust, and $\rho$ is the gas volume density.

Equations 2 and 3 are continuous in $v$, but for the purposes of computation we deal with photon groups whose properties are averaged over their entire range. We replace $N_{v}$ and $F_{v}$ with $N_{i}$ and $F_{i}$, which are the integrated sums 
over the range. The choice of photons groups for RAMSES$\mathrm{RT}$ is easily customised. Mainly we are concerned with four groups: 1, the $\mathrm{LW}$ band of $\mathrm{H}_{2}$-dissociating radiation $(11.2 \mathrm{eV}$ to $13.60 \mathrm{eV}) ; 2$, H I-ionising (13.60 eV to $24.59 \mathrm{eV}) ; 3$, He Iionising (24.59 eV to $54.42 \mathrm{eV}$ ); and 4, He II-ionising (54.42 $\mathrm{eV}$ and above) radiation. $\mathrm{H} \mathrm{I}$ and $\mathrm{H}_{2}$ are ionised by groups 2, 3, and 4; He I by groups 3 and 4; and He II by group 4 .

The pressure tensor, $\mathbb{P}_{\boldsymbol{v}}$, closes Equations 2 and 3 and is usually the product of the photon number density and the Eddington tensor, for which several approximations exist. We use the M1 closure relation (Levermore 1984), further details of which are given in Rosdahl et al. (2013).

Equations 2 and 3 are solved for each time step and photon group by an operator-splitting strategy. The photon flux and density, and species abundances are updated in a fixed order: photon injection, photon transport, and thermochemistry.

The photon injection step solves a single equation,

$\frac{\partial N_{i}}{\partial t}=\dot{N}_{i}^{\star}$

to account for all the photons injected into a cell, usually in galaxy simulations by stellar sources. This is carried out discretely over each photon group $i$, and sums over all the photon sources in the cell.

In the transport step the photons are treated as freeflowing between cells, described by the equations,

$$
\begin{aligned}
\frac{\partial N_{i}}{\partial t}+\nabla \cdot \mathbf{F}_{i} & =0 \\
\frac{\partial \mathbf{F}_{i}}{\partial t}+c^{2} \nabla \cdot \mathbb{P}_{i} & =0 .
\end{aligned}
$$

There are many functions available to solve these equations for the intercell flux. RAMSES-RT provides two options. The Harten-Lax-van Leer (HLL) flux function (Harten et al. 1983 ) is ideal for modelling beams and shadows, but shows asymmetries for isotropic sources. The Global Lax Friedrich (GLF) function ( $\operatorname{Lax} 1954)$ is better suited for isotropic sources and preserves symmetry, but tends to diffuse beams. Both these functions are useful depending on the scenario.

Finally, the thermochemistry step handles the interactions between the photons, gas temperature, dust, and the gas species $\mathrm{H}_{2}, \mathrm{H}$, $\mathrm{H}$ II, HeI, HeII, and HeIII. Equations 2 and 3 are solved without the injection or divergence terms. Non-equilibrium chemistry equations are too stiff to solve explicitly, due to timescales differing by orders of magnitude which renders the timesteps too small. Instead, we solve the chemistry semi-implicitly in a specific order based on the algorithm of Anninos et al. (1997). We will expand on this step in Section 2.4 after first introducing the details of $\mathrm{H}_{2}$ chemistry in Section 2.3

\subsection{Molecular hydrogen recipe}

In this section we describe in detail the new $\mathrm{H}_{2}$ chemistry implemented into RAMSES-RT. For the three major species of hydrogen the reaction rates are given by,

$$
\begin{aligned}
& \dot{n}_{\mathrm{H}}{ }_{\mathrm{II}}=-\dot{n}_{\mathrm{H}}-2 \dot{n}_{\mathrm{H}_{2}} \text {, } \\
& \dot{n}_{\mathrm{H} \mathrm{I}}=\alpha_{\mathrm{H}}(T) n_{\mathrm{e}} n_{\mathrm{H}}{ }_{\text {II }}-\beta_{\mathrm{H}, \mathrm{e}}(T) n_{\mathrm{e}} n_{\mathrm{H}} \\
& -\Gamma_{\mathrm{HI}_{\mathrm{I}}}\left(N_{\mathrm{HI}}\right) n_{\mathrm{H} \mathrm{I}}-2 \dot{n}_{\mathrm{H}_{2}} \text {, } \\
& \dot{n}_{\mathrm{H}_{2}}=\alpha_{\mathrm{H}_{2}}(T, Z) n_{\mathrm{H}} n_{\mathrm{H}_{\mathrm{I}}}-\beta_{\mathrm{H}_{2} \mathrm{H}_{\mathrm{I}}}(T) n_{\mathrm{H}_{\mathrm{I}}} n_{\mathrm{H}_{2}} \\
& -\beta_{\mathrm{H}_{2} \mathrm{H}_{2}}(T) n_{\mathrm{H}_{2}}^{2}-\Gamma_{\mathrm{H}_{2}}\left(N_{\mathrm{H}_{2}}\right) n_{\mathrm{H}_{2}}-\Gamma_{\mathrm{H}_{2}}^{+}\left(N_{\mathrm{H}_{\mathrm{I}}}\right) n_{\mathrm{H}_{2}} \text {, }
\end{aligned}
$$

where $n$ is the number density of the subscript species $\left(\mathrm{H}_{2}\right.$, $\mathrm{H} \mathrm{I}, \mathrm{H}$ II, or $e$ for electrons), $\alpha$ is the formation/recombination rate of the subscript species, $\beta$ the collisional dissociation/ionisation rate between the two subscript species, $\Gamma$ the photoionisation/dissociation rate of the subscript species, $N$ number density of photodissociating/ionising photon group(s) of the subscript species, and $T$ the temperature. $\mathrm{H}_{2}$ requires two photo-destruction terms: $\Gamma_{\mathrm{H}_{2}}\left(N_{\mathrm{H}_{2}}\right)$ for the dissociation by LW photons, and $\Gamma_{\mathrm{H}_{2}}^{+}\left(N_{\mathrm{H}_{\mathrm{I}}}\right)$ for photoionisation by the same photon groups that ionise H I. However, as we will explain in Section 2.3.1. we treat $\mathrm{H}_{2}$ ionisation as a dissociation that produces $\mathrm{HI}$ and not $\mathrm{HII}$. The rates for formation, collisional ionisation, and photoionisation of $\mathrm{H}$ I are preserved from Rosdahl et al. (2013). The $\mathrm{H}_{2}$ rate coefficients for formation, collisions, and photo-destruction are described in the following section.

\subsubsection{Molecular hydrogen rate coefficients}

We draw our $\mathrm{H}_{2}$ coefficients for the rate equations from a wide range of sources:

$$
\begin{aligned}
\alpha_{\mathrm{H}_{2}}(T, Z) & =\left(Z \frac{6.0 \times 10^{18} T_{0}^{0.5}}{1+0.4 T_{2}^{0.5}+0.2 T_{2}+0.08 T_{2}^{2}}\right. \\
& \left.+8.0 \times 10^{-19} T_{3}^{0.88}\right) \mathrm{cm}^{3} \mathrm{~s}^{-1}, \\
\beta_{\mathrm{H}_{2} \mathrm{H}}(T) & =\left(7.073 \times 10^{-19} T_{0}^{2.012}\right. \\
& \left.\times \frac{e^{-5.179 \times 10^{4} / T_{0}}}{\left(1+2.130 \times 10^{-5} T_{0}\right)^{3.512}}\right) \mathrm{cm}^{3} \mathrm{~s}^{-1}, \\
\beta_{\mathrm{H}_{2} \mathrm{H}_{2}}(T) & =\left(5.996 \times 10^{-30} T_{0}^{4.1881}\right. \\
& \left.\times \frac{e^{-5.466 \times 10^{4} / T_{0}}}{\left(1+6.761 \times 10^{-6} T_{0}\right)^{5.6881}}\right) \mathrm{cm}^{3} \mathrm{~s}^{-1}, \\
\Gamma_{\mathrm{H}_{2}}\left(N_{\mathrm{H}_{2}}\right) & =\sigma_{1 \mathrm{H}_{2}}^{N} c_{r} N_{1}, \\
\Gamma_{\mathrm{H}_{2}}^{+}\left(N_{\mathrm{H}_{\mathrm{I}}}\right) & =\sum_{i=2}^{M} \sigma_{i \mathrm{H}_{2}}^{N} c_{r} N_{i},
\end{aligned}
$$

where $T_{0}=\frac{T}{1 K}, T_{2}=\frac{T}{100 \mathrm{~K}}$, and $T_{3}=\frac{T}{1000 \mathrm{~K}} \cdot \sigma_{i \mathrm{H}_{2}}^{N}$ is the average destruction cross-section between species $i$ and $\mathrm{H}_{2}$, $M$ is the total number of photon groups, and the subscript 1 refers to the first photon group, which is the LW band in this paper. For $\mathrm{H}_{2}$ formation $\left(\alpha_{\mathrm{H}_{2}}\right)$ we use Draine \& Bertoldi (1996) and Hollenbach \& McKee (1979) for the catalysation with dust grains, and McKee \& Krumholz (2010) for gas phase formation. We only take into account the collisional destruction between $\mathrm{H}_{2}$ and $\mathrm{HI}_{\mathrm{I}}\left(\beta_{\mathrm{H}_{2} \mathrm{HI}}\right)$ (Dove \& Mandy 1986) and itself $\left(\beta_{\mathrm{H}_{2} \mathrm{H}_{2}}\right)$ (Martin et al. 1998) because these two collisions have the highest rates, and $\mathrm{H}_{2}$ is unlikely to exist with $\mathrm{H}$ II. For the photodissociation of $\mathrm{H}_{2}$ by LymanWerner photons $\left(\Gamma_{\mathrm{H}_{2}}\right)$ we use a cross-section derived from 
the photodissociation rate in Sternberg et al. (2014) and treat it as constant due to the the small range of the LW band,

$\sigma_{1 \mathrm{H}_{2}}^{N}=2.1 \times 10^{-19} \mathrm{~cm}^{2}$.

The ionisation of $\mathrm{H}_{2}$ occurs via a two step process (Abel et al. 1997). Firstly,

$\mathrm{H}_{2}+\gamma \rightarrow \mathrm{H}_{2}^{+}+\mathrm{e}^{-}$,

and secondly one of two processes occurs depending on the frequency of the incident photon:

$\mathrm{H}_{2}{ }^{+}+\gamma \rightarrow \mathrm{HI}+\mathrm{H} \mathrm{II}$,

$\mathrm{H}_{2}{ }^{+}+\gamma \rightarrow 2 \mathrm{H} \mathrm{II}+\mathrm{e}^{-}$.

However, to depict this entire chain of reactions realistically we would need to track the intermediate species $\mathrm{H}_{2}{ }^{+}$at an added computational cost. In order to keep our methodology simple, we model $\mathrm{H}_{2}$ ionisation as the following process:

$\mathrm{H}_{2}+\gamma \rightarrow 2 \mathrm{H} \mathrm{I}$,

$\mathrm{HI}+\gamma \rightarrow \mathrm{HII}+\mathrm{e}^{-}$.

Essentially we treat the ionisation of $\mathrm{H}_{2}$ as a dissociation. Because the wavelengths that ionise $\mathrm{H}_{2}$ and $\mathrm{H}$ I are virtually identical ( $h v \geq 15.42 \mathrm{eV}$ for $\mathrm{H}_{2}$ and $h v \geq 13.60 \mathrm{eV}$ for $\mathrm{H}_{\mathrm{I}}$ ) we assume that the $\mathrm{H}$ I produced from ionisation of $\mathrm{H}_{2}$ is quickly ionised into $\mathrm{H}$ II. We take the ionisation cross-section from Abel et al. (1997) for the first reaction in Equation 16 to be our ionisation cross-section for $\mathrm{H}_{2}$ ionisation:

$\sigma_{\mathrm{H}_{2}}^{+N}(v)= \begin{cases}0, & h v<15.42 \\ 6.2 \times 10^{-18} h v-9.4 \times 10^{-17}, & 15.42 \leq h v<16.50 \\ 1.4 \times 10^{-18} h v-1.48 \times 10^{-17}, & 16.50 \leq h v<17.7 \\ 2.5 \times 10^{-14}(h v)^{-2.71}, & 17.7 \leq h v,\end{cases}$

where the units for $\sigma_{\mathrm{H}_{2}}^{+N}(v)$ are in $\mathrm{cm}^{2}$ and $h v$ are in $\mathrm{eV}$. The continuous function, $\sigma_{\mathrm{H}_{2}}^{+N}(v)$, in Equation 21 is replaced in Equation 14 by discrete values, $\sigma_{i \mathrm{H}_{2}}^{N}$, that are the average cross-sections over each photon group, $i$.

\subsubsection{Molecular hydrogen self-shielding by line overlap}

RAMSES-RT already includes shielding for all species by destruction of the photons that ionise or dissociate gas. However, as described in our introduction, we need to enhance the destruction of LW photons due to $\mathrm{H}_{2}$ self-shielding processes. Here more photons are absorbed than $\mathrm{H}_{2}$ destroyed, and LW line overlap interferes with dissociation at higher column densities.

We take advantage of RAMSES-RT's unique position as a moment-based radiative transfer code. LW absorption is highly dependent on the photon's wavelength. We do not track individual bands of the LW photons, but as a group we can determine an overall reduction in photon number density because of $\mathrm{H}_{2}$ absorption. We introduce self-shielding in the destruction term for the photon density update to determine how many photons are absorbed by $\mathrm{H}_{2}$,

$D_{1 \mathrm{H}_{2}}=S_{1 \mathrm{H}_{2}}^{S} c_{r} \sigma_{1 \mathrm{H}_{2}}^{N} n_{\mathrm{H}_{2}}$,

where $D_{1 \mathrm{H}_{2}}$ is the destruction rate of LW photons, $S_{1 \mathrm{H}_{2}}^{S}$ is the self-shielding factor, and $\sigma_{1 \mathrm{H}_{2}}^{N}$ is the photodissociation cross-section between $\mathrm{H}_{2}$ and the LW photon band. As we will show in Section 3.3 a constant $S_{1 \mathrm{H}_{2}}^{s} \sim 500$ reproduces realistic self-shielding in a variety of environments, while being computationally expedient. Our method to enhance LW photo-destruction is in contrast to other codes (Gnedin \& Kravtsov 2011; Lupi et al. 2017, Katz et al. 2017) that calculate $\mathrm{H}_{2}$ self-shielding by decreasing $\mathrm{H}_{2}$ destruction.

Because we keep track of the photon density using a moment-based method, the LW band's cumulative reduction as it travels though an $\mathrm{H}_{2}$ region over multiple timesteps can be taken as it encountering a shielding column density; we need neither to convert our volume into a column density, nor to use a non-local method to calculate column density.

\subsection{Thermochemistry step}

Much of mechanics of the thermochemistry step is detailed in Rosdahl et al. (2013), with additions from Rosdahl \& Teyssier (2015). Here we emphasize our $\mathrm{H}_{2}$ addition to the formalism.

Over a time step, from $t$ to $t+\Delta t$, RAMSES-RT evolves the thermochemistry state in each cell given by $\mathcal{U}_{\mathcal{T}}=\left(\varepsilon, x_{\mathrm{H}}\right.$, $\left.x_{\mathrm{H} \text { II }}, x_{\mathrm{He} \mathrm{I}}, x_{\mathrm{He} \mathrm{I}}, N_{i}, \mathbf{F}_{i}\right)$, where $\varepsilon=E-\frac{1}{2} \rho \mathbf{u}^{2}$ is the thermal energy density. The non-equilibrium thermochemistry equations are too stiff to be solved expediently by an implicit solver, and instead are solved in a fixed order as inspired by Anninos et al. (1997). The order in which the equations are solved is: photon density and flux update, thermal update, hydrogen fraction update, and helium ionisation fraction update. At the end of each step, the quantity is checked to see if it has changed more than $10 \%$. If it has then there is no update and the procedure is run again with $0.5 \Delta t$. Once every quantity has been updated and the $10 \%$ change has not been violated, a final check is taken. If the change in $\mathcal{U}_{\mathcal{T}}$ is less than $5 \%$ then the next time step will be $2 \Delta t$.

The following subsections detail each quantity in the thermochemistry step. The equations are given for case A recombination, but if OTSA is used then case B recombination rates would replace them.

\subsubsection{Photon density and flux update}

The photon density, $N$, and flux, $F$, are updated by each photon group, $i$, individually since they operate independently of each other. They are given by,

$$
\begin{aligned}
\frac{\partial N_{i}}{\partial t} & =\dot{N}_{i}+C_{i}-N_{i} D_{i}, \\
\frac{\partial F_{i}}{\partial t} & =\dot{F}_{i}-F_{i} D_{i},
\end{aligned}
$$

where $\dot{N}_{i}$ is the change in photon density from the RT transport solver, $C_{i}$ is the photon creation from recombination, $D_{i}$ is photon destruction from absorption terms, and $\dot{F}_{i}$ is the change in photon flux. There is no corresponding creation term for the flux because radiation from recombination is assumed to be spherically symmetric. 
Creation and destruction are given by,

$$
\begin{aligned}
C_{i} & =\sum_{j}^{\mathrm{H} \mathrm{II}, \mathrm{He} \text { I, Не II }} b_{j i}^{r e c}\left(\alpha_{j}^{A}-\alpha_{j}^{B}\right) n_{j} n_{e}, \\
D_{i} & =\kappa_{d} \rho Z f_{d} c_{r}+A_{i}^{P E}+\sum_{j}^{\mathrm{H}_{2}, \mathrm{HI}, \mathrm{He} \text { I, Не II }} S_{i j}^{S} c_{r} \sigma_{i j}^{N} n_{j},
\end{aligned}
$$

where $b_{j i}^{r e c}$ is a boolean to describe the photon group that the $j$-species recombines into, $\alpha_{j}^{A}(T)$ and $\alpha_{j}^{B}(T)$ are the case $\mathrm{A}$ and $\mathrm{B}$ recombination rates, $n_{j}$ is the number density of gas $j, n_{e}$ is the number density of electrons, $\kappa_{d}$ is the dust opacity, $\rho$ is the gas volume density, $f_{d}$ is the fraction of gas that holds dust, $c_{r}$ is the reduced speed of light, and $\sigma_{i j}^{N}$ is the destruction cross-section between gas species $j$ and photon group $i$. $S_{i j}^{S}$ is the self-shielding factor for $\mathrm{H}_{2}$ as described in Section 2.3 to boost the destruction of LW photons. If the photon species is $\mathrm{LW}$ and the gas species is $\mathrm{H}_{2}$ then $S_{1 \mathrm{H}_{2}}^{S}=500$; otherwise $S_{i j}^{S}=1 . A_{i}^{P E}$ is the the absorption term from the photoelectric effect added in Butler et al. (2017) and lifted from Forbes et al. (2016), which is only non-zero in the LW band:

$A_{1}^{P E}=5.36 \times 10^{-23} \mathrm{~cm}^{2} c_{r} n_{H} Z e^{-T / 2 \times 10^{3} \mathrm{~K}}$.

The exponential factor prevents the photoelectric effect from having an impact at temperatures beyond which the dust is heated into non-existence (Guhathakurta \& Draine 1989). The photoelectric effect occurs over energies 8 to $13.6 \mathrm{eV}$, which goes a little lower than the LW band, but we do not add an extra photon group for computational expediency.

If OTSA is on then there is no creation term (Equation 25 because the photons are assumed to be immediately reabsorbed. $\mathrm{H}_{2}$ formation by dust does not involve the emission of photons, and while the gas phase formation does, its rate is much too weak to have an impact on our simulations, and therefore the $C_{i}$ term does not involve any photons from $\mathrm{H}_{2}$ creation.

Photon density and flux advance in time with a partly semi-implicit Euler formulation given by,

$N_{i}^{t+\Delta t}=\frac{N_{i}^{t}+\Delta t\left(\dot{N}_{i}+C_{i}\right)}{1+\Delta t D_{i}}$,

$F_{i}^{t+\Delta t}=\frac{F_{i}^{t}+\Delta t \dot{F}_{i}}{1+\Delta t D_{i}}$

At the end of this step momentum is transferred from the photons to the gas and energy absorbed by dust is added to the IR photons if this group is in use, as outlined in Rosdahl \& Teyssier (2015).

\subsubsection{Thermal update}

For each gas cell in RAMSES-RT, the temperature can be obtained via,

$T=\varepsilon \frac{(\gamma-1) m_{H}}{\rho k_{\mathrm{B}}} \mu$,

where $\varepsilon$ is the thermal energy density, $\gamma$ is the ratio of specific heats, $m_{H}$ is the proton mass, $k_{\mathrm{B}}$ is the Boltzman constant, and $\mu$ is the average gas particle mass in units of $m_{H}$.
However, because $\mu$ depends on the ionisation fraction, $T_{\mu}=T / \mu$ is evolved instead of $T$ via,

$$
\begin{aligned}
& \frac{\partial T_{\mu}}{\partial t}=\frac{(\gamma-1) m_{H}}{\rho k_{B}}(\mathcal{H}-\mathcal{L}), \\
& \mathcal{H}=\sum_{j}^{\mathrm{H}_{2}, \mathrm{H \textrm {I }}, \mathrm{He} \mathrm{I}, \mathrm{He} \text { II }} n_{j} \sum_{i=1}^{M} c_{r} N_{i}\left(\bar{\epsilon}_{i} \sigma_{i j}^{E}-\epsilon_{j} \sigma_{i j}^{N}\right) \\
& +\mathcal{H}_{P E} \text {, } \\
& \mathcal{L}=\left[\zeta_{\mathrm{H}_{\mathrm{I}}}(T)+\psi_{\mathrm{H}}(T)\right] n_{e} n_{\mathrm{H}} \\
& +\zeta_{\mathrm{HeI}}(T) n_{e} n_{\mathrm{He} \mathrm{I}} \\
& +\left[\zeta_{\mathrm{He} \text { II }}(T)+\psi_{\mathrm{He} \mathrm{II}}(T)+\eta_{\mathrm{He} \mathrm{II}}^{\mathrm{A}}(T)\right. \\
& \left.+\omega_{\mathrm{He} \mathrm{II}}(T)\right] n_{e} n_{\mathrm{He} \mathrm{II}} \\
& +\eta_{\mathrm{H} \text { II }}^{\mathrm{A}}(T) n_{e} n_{\mathrm{H} \mathrm{II}} \\
& +\eta_{\mathrm{He} \mathrm{III}}^{\mathrm{A}}(T) n_{e} n_{\mathrm{He} \mathrm{III}} \\
& +\theta(T) n_{e}\left(n_{\mathrm{H} \text { II }}+n_{\mathrm{He} \text { II }}+4 n_{\mathrm{He} \mathrm{III}}\right) \\
& +\varpi(T) n_{e} \\
& +\Lambda_{Z}(T) \\
& +\Lambda_{\mathrm{H}_{2}}(T) \text {. }
\end{aligned}
$$

In the heating term, $\mathcal{H}, \bar{\epsilon}_{i}$ is the photon average energy, $\epsilon_{j}$ is the photodestruction energy, $\sigma_{i j}^{N}$ is the average crosssection between group $i$ and species $j$, and $\sigma_{i j}^{E}$ is the energy weighted cross-section. In simulations with star particles, these are calculated from SED tables. For this paper, we do not work with stars and instead use the cross-sections averaged over a black body. $\mathcal{H}_{P E}$ is the heating from the photoelectric effect, as given in Forbes et al. (2016):

$\mathcal{H}_{P E}=8.5 \times 10^{-26} \mathrm{erg} \mathrm{s}^{-1} f_{\mathrm{Hab}} n_{H} Z e^{-T / 2 \times 10^{3} \mathrm{~K}}$,

$f_{\mathrm{Hab}}=\epsilon_{1} N_{1} c_{r} /\left(1.5859 \times 10^{-3} \mathrm{erg} \mathrm{s}^{-1} \mathrm{~cm}^{-2}\right)$,

where $\epsilon_{1}$ is the energy of group 1 in ergs, and $f_{H a b}$ normalises our field to the Habing value (Habing 1968).

The cooling term, $\mathcal{L}$, includes collisional ionisation $\zeta$, collisional excitation $\psi$, recombination $\eta$, dielectronic recombination $\omega$, Bremsstrahlung $\theta$, and Compton cooling $\varpi$. Their functional forms and sources are given in Rosdahl et al. (2013). $\Lambda_{Z}$ is the contribution of metals to cooling, from tables generated by CLOUDY above $10^{4} \mathrm{~K}$, and below $10^{4} \mathrm{~K}$ using the fine structure cooling rates from Rosen \& Bergman (1995) (Rosdahl et al. 2017).

Below temperatures of $5000 \mathrm{~K}, \mathrm{H}_{2}$ is the dominant coolant (Gnedin \& Kravtsov 2011). We use a cooling functional from similar to Halle \& Combes (2013) for $\mathrm{H} \mathrm{I}_{-} \mathrm{H}_{2}$ and $\mathrm{H}_{2}-\mathrm{H}_{2}$ collisional cooling only in the low density limit $(n \rightarrow 0)$, because our galactic simulations will not resolve high enough densities to reach local thermal equilibrium rates (LTE),

$\Lambda_{\mathrm{H}_{2}}(T)=\Lambda_{\mathrm{H}_{2} \mathrm{H}(n \rightarrow 0)}(T) n_{\mathrm{H}_{\mathrm{I}}} n_{\mathrm{H}_{2}}+\Lambda_{\mathrm{H}_{2} \mathrm{H}_{2}(n \rightarrow 0)}(T) n_{\mathrm{H}_{2}}^{2}$,

where $\Lambda_{\mathrm{H}_{2} \mathrm{H} \mathrm{I}(n \rightarrow 0)}$ and $\Lambda_{\mathrm{H}_{2} \mathrm{H}_{2}(n \rightarrow 0)}$ are the low-density limits of the $\mathrm{H}_{2}$ collisional cooling coefficients from Hollenbach \& McKee (1979) in units of $\mathrm{cm}^{3} \mathrm{erg} / \mathrm{s}$.

The temperature is then updated semi-implicitly using the updated values for photon density and flux from Section 2.4.1 but the un-updated values for the hydrogen and helium species:

$T_{\mu}^{t+\Delta t}=T_{\mu}^{t}+\frac{\Lambda K \Delta t}{1-\Lambda^{\prime} K \Delta t}$. 
Here $\Lambda \equiv \mathcal{H}-\mathcal{L}, \Lambda^{\prime} \equiv-\frac{\partial \mathcal{L}}{\partial T_{\mu}}$, and $K \equiv \frac{(\gamma-1) m_{H}}{\rho k_{B}}$.

\subsubsection{Species fraction update}

We only store the variables $x_{\mathrm{H}_{\mathrm{I}}}$ and $x_{\mathrm{H} \text { II }}$, and recover $x_{\mathrm{H}_{2}}$ via $x_{\mathrm{H}_{2}}=0.5\left(1-x_{\mathrm{H}}-x_{\mathrm{H}}\right)$. However, all three quantities are evolved in order to ensure consistency and stability.

These fractions evolve as,

$$
\begin{aligned}
& \frac{\partial x_{\mathrm{H}_{2}}}{\partial t}=x_{\mathrm{H}_{\mathrm{I}}} \alpha_{\mathrm{H}_{2}} n_{\mathrm{H}} \\
& -x_{\mathrm{H}_{2}}\left(\beta_{\mathrm{H}_{2} \mathrm{H} \mathrm{I}_{\mathrm{I}}} n_{\mathrm{H}}-\beta_{\mathrm{H}_{2} \mathrm{H}_{2}} n_{\mathrm{H}_{2}}-\sum_{i=1}^{M} \sigma_{i \mathrm{H}_{2}}^{N} c_{r} N_{i}\right),
\end{aligned}
$$

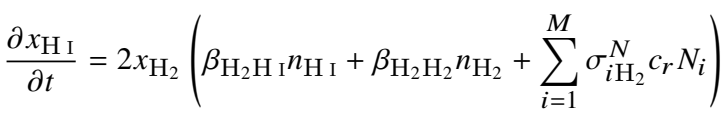

$$
\begin{aligned}
& +x_{\mathrm{H} \mathrm{II}} \alpha_{\mathrm{H} \text { II }}^{A} n_{\mathrm{e}} \\
& -x_{\mathrm{H} \text { I }}\left(2 \alpha_{\mathrm{H}_{2}} n_{\mathrm{H}}-\beta_{\mathrm{H} \mathrm{I}} n_{\mathrm{e}}+\sum_{i=1}^{M} \sigma_{i \mathrm{H}_{\mathrm{I}}}^{N} c_{r} N_{i}\right) \text {, }
\end{aligned}
$$

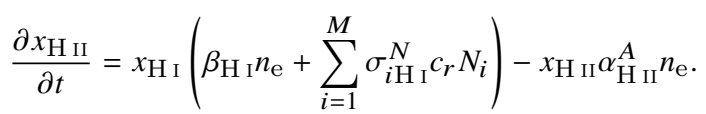

The respective destruction and creation coefficients corresponding to $\mathrm{H}_{2}$ are given in Section 2.3.1 while the coefficients for $\mathrm{H}$ I and $\mathrm{H}$ II are given in Rosdahl et al. (2013). Each of these Equations 38 to 40 for a species faction $x$ may be reformulated as,

$$
\frac{\partial x}{\partial t}=C-x D
$$

for their creation term $C$ and destruction $D$. We then update each species fraction in order of $\mathrm{H}_{2}, \mathrm{HI}$, and $\mathrm{H}$ II using the semi-implicit method,

$x^{t+\Delta t}=\frac{x^{t}+C \Delta t}{1+D \Delta t}$.

This expression always uses the updated values of $N_{i}$ and $T_{\mu}$ from Sections 2.4.1 and 2.4.2 The $\mathrm{H}_{2}$ update uses entirely un-updated values of the species fractions. The H I update uses the new value for $\mathrm{H}_{2}$, while all other species fractions are un-updated. Finally, the H II update uses the new H I fraction.

At the end of this step, we enforce conservation of hydrogen, by checking that $2 x_{\mathrm{H}_{2}}+x_{\mathrm{H}}+x_{\mathrm{H} \text { II }}=1$, and when this fails we lower the largest fraction accordingly.

Updating the fraction of helium species, between He I, HeII, and HeIII, follows an almost identical procedure as above and is unchanged from Rosdahl et al. (2013). We do not cover helium in this paper.

\section{THE TESTS}

Rosdahl et al. (2013) uses the Iliev series of benchmark tests (Iliev et al. 2006, 2009) for radiative transfer codes in atomic and ionised environments to verify its robustness. It is difficult to create tests with analytical solutions for radiative transfer codes, and so instead radiative transfer codes are compared to each other in these benchmark tests. If many codes agree, then they are taken to be correct.
For $\mathrm{H}_{2}$ formation in galaxy codes, however, there is no series of benchmark tests. Instead, we compare our code to PDR codes optimised for smaller scales. Our strategy is to begin with simple zero-dimensional tests, and add increasing complexity. In zero dimensions, that is a single cell, we can compare to an analytic solution. For one dimension, we can compare to more detailed PDR codes that are specialised for these scales, and extrapolate the results to two and three dimensions. Finally, for three dimensions we introduce a Strömgren sphere for an ionised hydrogen front in a neutral medium shell protecting a larger molecular medium. Our aim is to test our methodology against known solutions where they exist, and ensure sensible outcomes where there are no known solutions.

All these tests use only hydrogen with helium off, and frozen hydrodynamics. For the LW band we use groups 1, $11.2 \leq h v \leq 13.6 \mathrm{eV}$, and for $\mathrm{H}_{2}$ and $\mathrm{H}$ i ionisation radiation we use group 2, $13.6 \leq h v \leq 24.59 \mathrm{eV}$. Higher energies also ionise hydrogen, but their cross-sections are small enough to not have an impact.

\subsection{Single cell convergence}

These zero-dimensional tests are similar to those run in Rosdahl et al. (2013) to see if our method for $\mathrm{H}_{2}$ thermochemistry makes sense in simple situations. For all scenarios it is important to test for smoothness of evolution and if the final state is physically sensible. We evolve single cells with a homogeneous radiation-gas fluid. They have a range of hydrogen densities, initial temperatures, and initial atomic/ionised fractions. Density is fixed while hydrogen fractions evolve over time. Metallicity is fixed at the Solar value. We run each cell for $2 \times 10^{2} \mathrm{Myr}$, which is a little longer than the possible lifetime for molecular clouds from 30 to 100 Myr (Zasov \& Kasparova 2014).

There are four scenarios: with and without a fixed UV radiation field and with a fixed temperature or variable temperature. In the fixed temperature cases, we need to see if the cell's hydrogen fractions evolve to the equilibrium value. We obtain the equilibrium value by numerically iterating over the rate Equations 7, 8, and 9 until a steady state is reached.

For each scenario we test a grid of six fixed hydrogen densities $\left(10^{-4} \mathrm{~cm}^{-3} \leq n_{H} \leq 10^{6} \mathrm{~cm}^{-3}\right)$ and five fixed/initial temperatures $\left(10 \mathrm{~K} \leq T \leq 10^{7} \mathrm{~K}\right)$. In addition, for each density and temperature combination we test initial fractions of $x_{\mathrm{H}}=1.0,0.8,0.5,0.2$, and 0.0. The initial molecular fraction is always zero. The UV field is calculated from the $z=0$ Haardt and Madau background (Haardt \& Madau 1996) over the $\mathrm{H}_{2}$-dissociating and $\mathrm{H}$ I-ionising photon groups at redshift zero (photoionisation: $\Gamma_{\mathrm{UVH}_{2}}=$ $2.6 \times 10^{-18} \mathrm{~s}^{-1}$ and $\Gamma_{\mathrm{UVH}}=3.6 \times 10^{-14} \mathrm{~s}^{-1}$; and photoheating $\mathcal{H}_{\mathrm{UVH}_{2}}=1.8 \times 10^{-30} \mathrm{erg} \mathrm{s}^{-1}$ and $\mathcal{H}_{\mathrm{UVH}}=2.4 \times 10^{-25} \mathrm{erg}$ $\left.\mathrm{s}^{-1}\right)$.

We begin with fixed temperature and no UV background radiation (Figure 1) and compare the evolution to the equilibrium state for each density and temperature. In this situation, given any temperature the equilibrium state is the same for all densities. As expected, higher temperatures of $3.2 \times 10^{5} \mathrm{~K}$ and over lead to an ionised equilibrium state while intermediate $10^{4} \mathrm{~K}$ yields atomic, and temper- 
atures at $3.2 \times 10^{2} \mathrm{~K}$ and lower lead to a molecular state. In lower density environments below $10^{4} \mathrm{~K}$, from $10^{-4} \mathrm{~cm}^{-3}$ to $1 \mathrm{~cm}^{-3}$, the cell does not reach a fully molecular equilibrium state within the simulation time. By contrast, as the density increases the cells reach the equilibrium state much more quickly and $\mathrm{H}_{2}$ can form. In their work on molecular cloud simulations, Glover \& Mac Low (2007) show that in non-turbulent clouds with initial densities of $10 \mathrm{~cm}^{-3}, \mathrm{H}_{2}$ forms in about $10 \mathrm{Myr}$. Figure 1 supports this.

Next, we run the same test again but with a UV background (Figure 2). For the same temperatures and densities, the gas is more ionised and less molecular than without a UV background. Now the equilibrium state does depend on both density and temperature. This is because previously we only had the collisional destruction rates that are proportional to the density and so they cancelled out in the equilibrium calculations, while the destruction rate from the UV background is density independent. $3.2 \times 10^{5} \mathrm{~K}$ and higher yields an entirely ionised state for all densities. At $10^{4} \mathrm{~K}$, the gas is fully ionised at $10^{-4} \mathrm{~cm}^{-3}$. At $100 \mathrm{~cm}^{-3}$, the final fraction is mostly $\mathrm{HI}$, with traces of $\mathrm{HII}$, and higher densities are entirely atomic. At lower temperatures, $3.2 \times 10^{5} \mathrm{~K}$ and below, our final states are only fully molecular at densities of 100 $\mathrm{cm}^{-3}$ and higher. At $10^{-2} \mathrm{~cm}^{-3}$ the final state is a $\mathrm{HI}$ and $\mathrm{H}$ II mix, while at lower densities the cell is almost entirely ionised. Our $10^{-2}$ and $1 \mathrm{~cm}^{-3}$ cells at these lower temperatures do not reach the equilibrium state in the simulation time.

The next permutation is to allow for a variable temperature, again with the same range of densities and initial temperatures. We rerun this first without a UV background (Figure 3). In these conditions it is clear that little cooling occurs in the lowest density environment, $10^{-4} \mathrm{~cm}^{-3}$. However as density is increased, the cooling increases for each initial temperature state. By $10^{2} \mathrm{~cm}^{-3}$ and higher, every initial temperature state cools quickly to a $\sim 10 \mathrm{~K}$ floor below which a cell will not cool any further. This is due to metal cooling (Section 2.4.2). The initially $10 \mathrm{~K}$ cells change little because they are already at this floor. The necessity of $\mathrm{H}_{2}$ for cooling is clear for the $3.2 \times 10^{5} \mathrm{~K}$ cases and higher, where the cooling begins to be affected by the initial H I fraction. The initial entirely atomic states cool the fastest, being the easiest to convert to $\mathrm{H}_{2}$, while increasing the initial ionisation fraction slows cooling. A certain density of $\mathrm{H}_{2}$ is reached before cooling begins to accelerate.

The picture is different when we use a UV background (Figure 4). Here the floor is density dependent, and lower for higher densities. $10^{-4} \mathrm{~cm}^{-3}$ cells of different initial ionisation factions do not reach the same temperature within the simulation time. The $10^{-2} \mathrm{~cm}^{-3}$ cells all have a $\sim 10^{4} \mathrm{~K}$ floor. Cells with initial temperatures below $10^{4}$ will heat up towards it. $1 \mathrm{~cm}^{-3}$ cells cool/heat to on order of $\sim 100 \mathrm{~K}$ and $100 \mathrm{~cm}^{-3}$ cells cool/heat to a little above our $\sim 10 \mathrm{~K}$ floor. Cells $10^{4} \mathrm{~cm}^{-3}$ and denser cool to $\sim 10 \mathrm{~K}$. We can compare this scenario to the fixed temperature case with UV (Figure 2 where molecular hydrogen does not form at all at the lower densities, and even at $10 \mathrm{~K}$ needs $100 \mathrm{~cm}^{-3}$ to form quickly in significant enough quantities within the simulation time. There are some small oscillations in the $10^{4} \mathrm{~cm}^{-3}$ column as the temperature hits $\sim 10 \mathrm{~K}$, due to equation stiffness, but these soon dissipate.

These tests are in line with the equilibrium values for the fixed temperature cases and for the variable temperature cases the results are reasonable. We can conclude that our thermochemistry is robust in zero dimensions.

\subsection{Self-shielding calibration}

It is important to calibrate our self-shielding factor in Equation 22 to realistically reproduce the transition depth between $\mathrm{HI}_{\mathrm{I}}$ and $\mathrm{H}_{2}$. We set up a series of one-dimensional simulations where a constant source of LW radiation travels through a low density region and hits a high-density, $\mathrm{H}_{2}$ region. The high-density region is fixed at $n=1,10,100$, and $1000 \mathrm{~cm}^{-3}$ for fluxes $0.1,1$, and $10 \chi$, fixed temperature at $50 \mathrm{~K}$ (chosen for consistency with Roellig et al. (2007)), and Solar metallicity. $\chi$ is the Draine flux, $\left(1.4 \times 10^{8}\right.$ photons $\mathrm{cm}^{-2} \mathrm{~s}^{-1}$ in the LW band), the standard UV background for the ISM (Draine 1978).

The top plot in Figure 5 shows the transition between $\mathrm{HI}$ and $\mathrm{H}_{2}$ in the high density region, without any selfshielding due to LW absorption line overlap. To convert from column density, $\mathcal{N}_{H}$, to visual extinction, $A_{V}$, we use the conversion, $A_{V}=6.289 \times 10^{-22} \mathcal{N}_{H}$ in order to be consistent with Roellig et al. (2007). We also plot the transition's location as predicted by Bialy \& Sternberg (2016), who give an analytic expression for the column density of transition between $\mathrm{H}$ I and $\mathrm{H}_{2}$ based on the Sternberg et al. (2014) theory for PDR regions:

$$
\begin{aligned}
\mathcal{N}_{\text {trans }} & =0.7 \ln \left[\left(\frac{\alpha G}{2}\right)^{1 / 0.7}+1\right] \times\left(1.9 \times 10^{-21} \mathrm{Zcm}^{2}\right)^{-1}, \\
\alpha G & =0.59 F_{L W_{\chi}}\left(\frac{100 \mathrm{~cm}^{-3}}{n_{H}}\right)\left(\frac{9.9}{1+8.9 Z}\right)^{0.37},
\end{aligned}
$$

where $\mathcal{N}_{\text {trans }}$ is the transition column density between $\mathrm{H}_{2}$ and $\mathrm{HI}, F_{L W \chi}$ is the incident LW flux in units of $\chi$, and $\alpha G$ is a dimensionless parameter for the dust optical depth. Without any self-shielding in our model, the dissociating LW photons penetrate too deeply into the high density region and too much $\mathrm{H}$ i forms as compared to Equation 43 . The general trends, however, are correct. Lower densities and higher fluxes yield deeper transitions.

We use Equation 43 to calibrate our $\mathrm{H}_{2}$ line overlap self-shielding model, and test a range of self-shielding factors to find the optimal value. The middle plot in Figure 5 shows that a constant $S_{1 \mathrm{H},}^{S} \sim 500$ boost to the destruction of LW radiation (Equation 22 gives a realistic match to the analytical transition point, especially for lower densities. It is less accurate for higher densities and at smaller visual extinctions. However, our application for this is full galaxy simulations where we do not resolve distances this small and the grid cell would be effectively entirely molecular at this density. We run the test again with the temperature allowed to vary (bottom of Figure 5) and find the match between the analytic expression and our model to be close.

We explore metallicity dependence in Figure 6 , by rerunning the same densities and fluxes with variable temperature and self-shielding, but with ten times and a tenth of Solar metallicity. In the higher metallicity case, we exclude $1000 \mathrm{~cm}^{-3}$ because the extremely small scales of the transition region. Here our transition and Equation 43 are spot on aside from the lowest transition column densities. In the low metallicity case, we tend to slightly over predict the size of the molecular region in the high column density cases, 

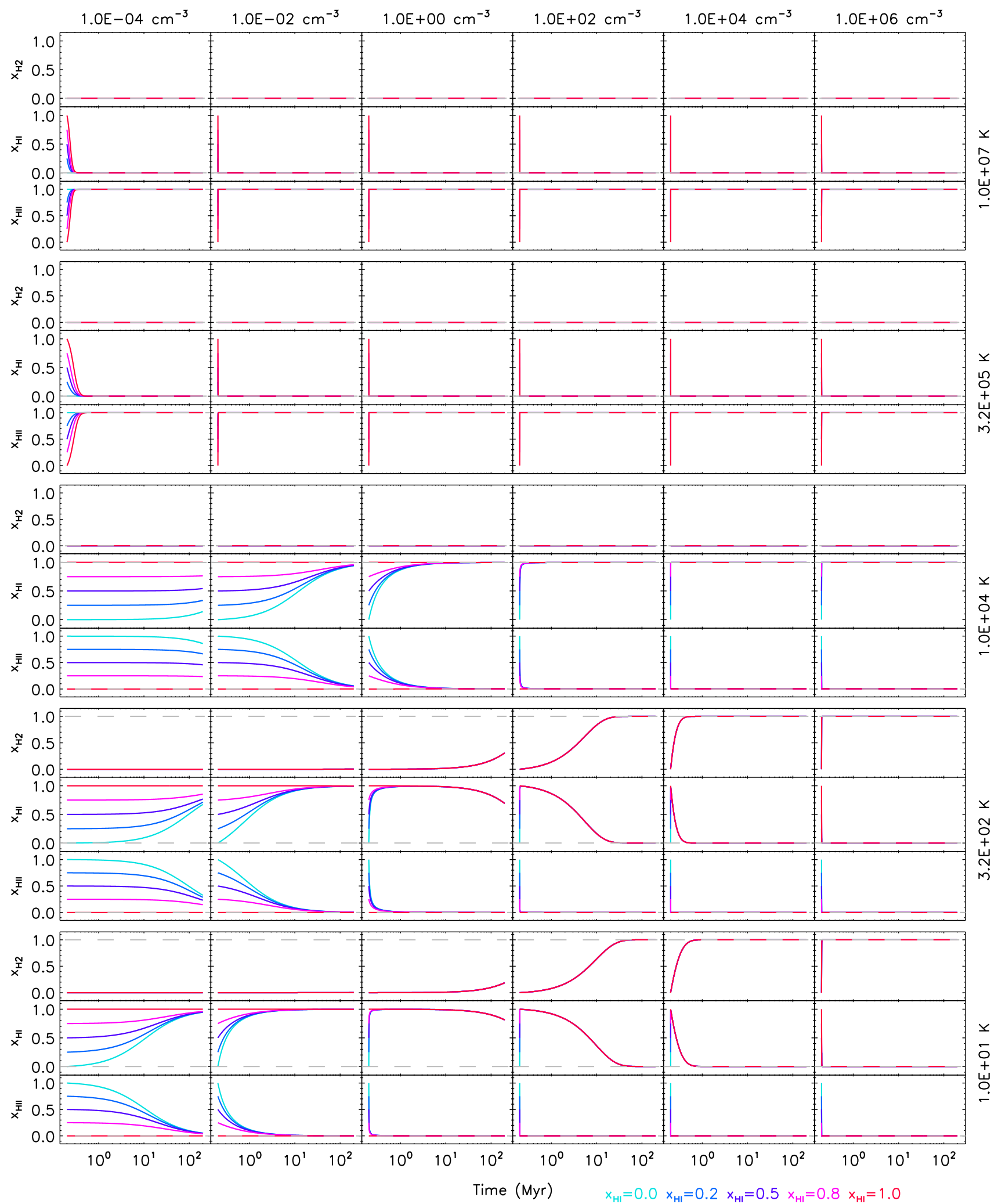

Figure 1. Hydrogen species evolution in zero-dimensional convergence tests for a range of fixed densities and temperatures. Coloured lines refer to different initial atomic hydrogen fractions. Grey dashed lines are the equilibrium states. 

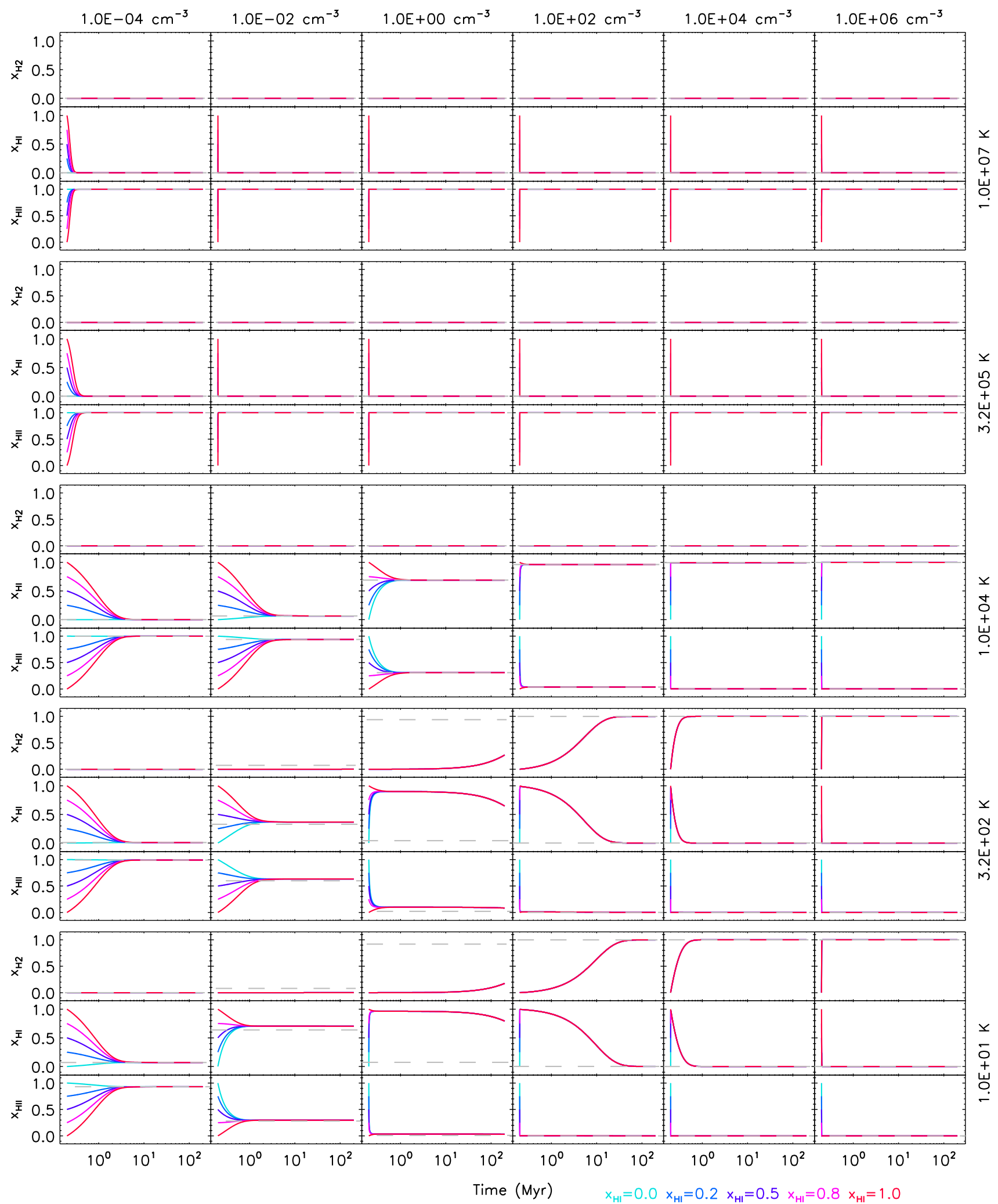

Figure 2. Hydrogen species evolution in zero-dimensional convergence tests for a range of fixed densities and temperatures. UV background is on. Coloured lines refer to different initial atomic hydrogen fractions. Grey dashed lines are the equilibrium states. 


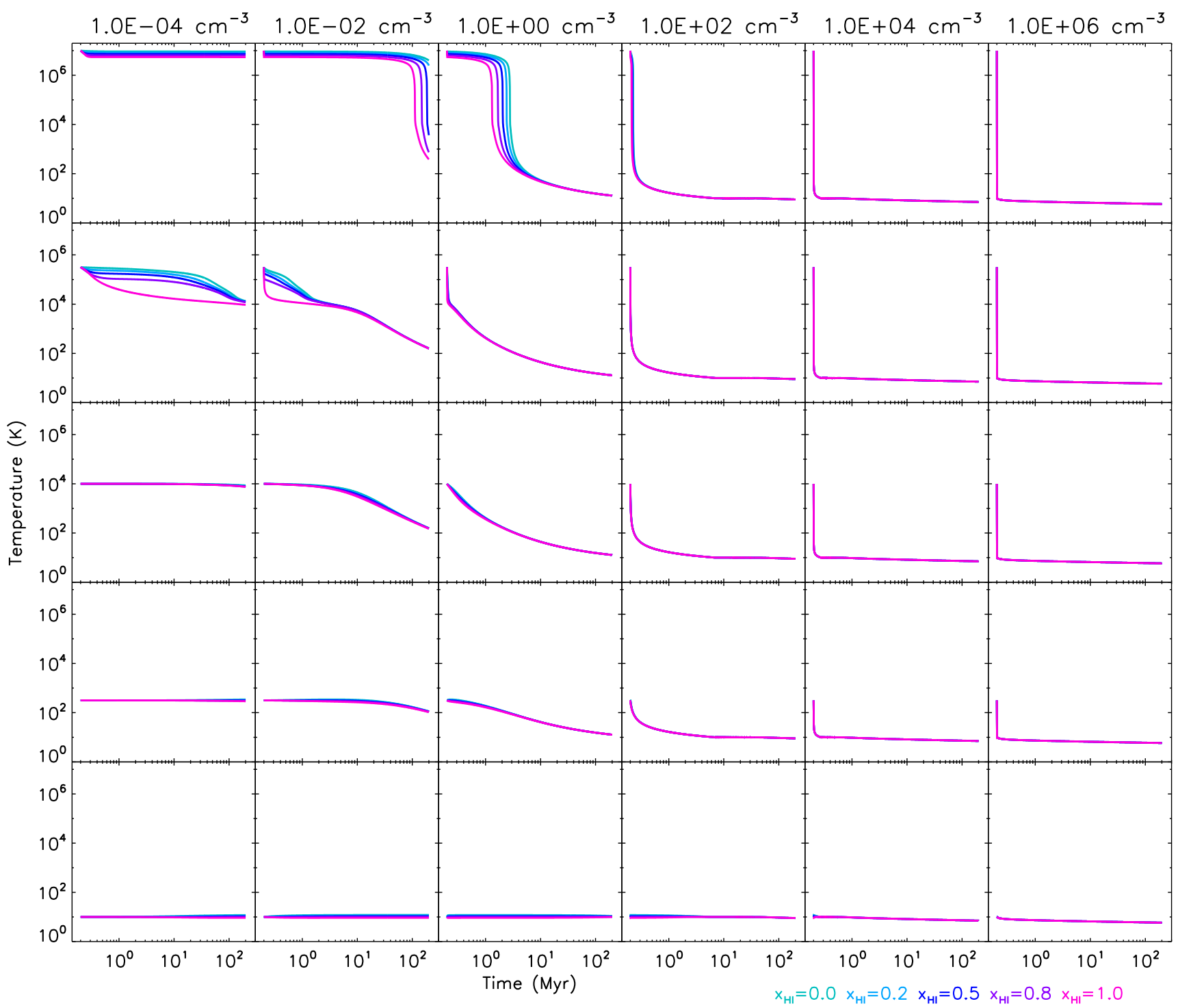

Figure 3. Temperature evolution in zero-dimensional tests for a range of fixed densities and initial temperatures. Coloured lines refer to different initial atomic hydrogen fractions. Initial molecular fraction is always zero.

and more closely predict the low column density transitions. In both of these extreme metallicity situations our transition point follows the correct trend where the $\mathrm{H}_{2}$ region is thicker for higher metallicity and thinner for lower metallicity, and we predict the transition depth closely enough for the purposes of galaxy simulations.

As a side note, when we experiment with self-shielding factors that use the cell size to calculate a column density for the Draine \& Bertoldi (1996) equation (such as the method found in Katz et al. (2017)), we find that the transition depth depends on the simulation resolution. This is undesirable since the transition should be independent of the grid's refinement level. The method we outline here in Equation 22 is robust against different resolutions.

\subsection{One-dimensional photodissociation regions}

Roellig et al. (2007) presents a series of benchmark tests for 10 PDR codes, not to mimic any specific astrophysical scenario but instead as a reference by which to understand present and compare future PDR models. The codes they use are CLOUDY (Ferland et al. 1998), COSTAR (Kamp \& Bertoldi 2000), нтвкW (Tielens \& Hollenbach 1985), KosmA- $\tau$ (Stoerzer et al. 1996), LEE96 (Lee et al. 1996), LEIDEN (Black \& van Dishoeck 1987), MeiJERINK (Meijerink \& Spaans 2005), MEudon (Le Bourlot et al. 1993), Sternberg (Sternberg \& Dalgarno 1989), and UCL_PDR (Taylor et al. 1993). In these benchmark tests a plane-parallel, one-dimensional, optically thick $\mathrm{H}_{2}$ slab is illuminated uni-directionally by a constant LW flux. They tested eight scenarios: $n=10^{3}$ and $10^{5.5} \mathrm{~cm}^{-3}, F_{L W}=10$ and $10^{5} \chi$, with temperature fixed at $50 \mathrm{~K}$ and variable.

We compare our model to these benchmark tests for the $n=10^{3} \mathrm{~cm}^{-3}$ cases. We do not test the $n=10^{5} \mathrm{~cm}^{-3}$ cases because of the extremely high resolution required to resolve the thin $\mathrm{HI}_{\mathrm{I}} \mathrm{H}_{2}$ transition layer, which our code is not spe- 


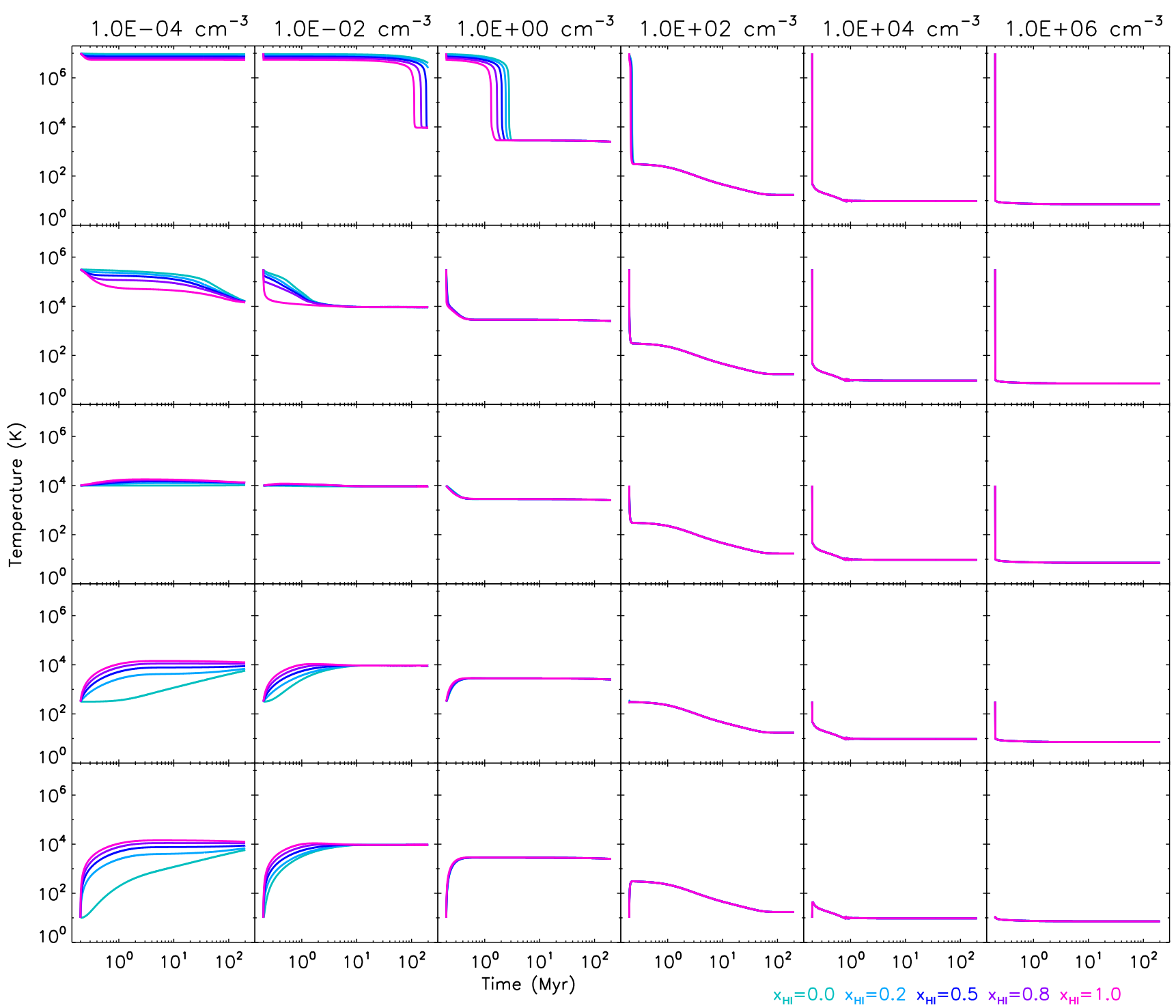

Figure 4. Temperature evolution in zero-dimensional tests for a range of fixed densities and initial temperatures. UV background is on. Coloured lines refer to different initial atomic hydrogen fractions. Initial molecular fraction is always zero.

cialised to do. At such high densities, the region is essentially entirely molecular. We use Solar metallicity for these tests. The boxsize is $10 \mathrm{pc}$ with the AMR grid resolution between $256^{1}$ and $16384^{1}$ cells.

Figure 7 shows the high density region profiles of the number density of $\mathrm{HI}$ and $\mathrm{H}_{2}$ and the LW photodissociation rate, for the $F_{L W}=10$, and $10^{5} \chi$ cases and a fixed $T=50 \mathrm{~K}$. The most striking feature is that our transition between atomic and molecular happens more abruptly in our model as compared to the Roellig et al. (2007) benchmark tests. In both the density and the photodissociation rate profiles we do not reproduce the gradual transition. This is expected due to a difference in how we handle $\mathrm{H}_{2}$ selfshielding. The traditional Draine (Draine \& Bertoldi 1996) and Draine-inspired functions follow a power law, while our constant factor leads to an exponential cut off. Nevertheless, this is incidental on the scales we will use for our galaxy simulations. We mainly seek to reproduce the transition depth, which is close enough for our purposes. A further feature is that in our model the atomic fraction drops to zero in the molecular region, while in Roellig et al. (2007) there is some residual $\mathrm{HI}$. This is due to the PDR codes' use of cosmic rays that can penetrate the molecular region and dissociate it, while our model does not include cosmic rays.

Figure 8 gives the same profiles as Figure 7. except with the addition of the temperature profile for the variable temperature case. Here our transition between atomic and molecular happens much more closely to that of the Roellig et al. (2007) benchmark tests, while the abruptness in transition shape remains. The cooling of the high-density region is also more abrupt in our model, following our exponential model for self-shielding as explained above. This highlights how $\mathrm{H}_{2}$ dominates the cooling process, as seen also in the single cells in Section 3.1 In our high flux case, $10^{5} \chi$, the atomic region is slightly hotter compared to the benchmark 

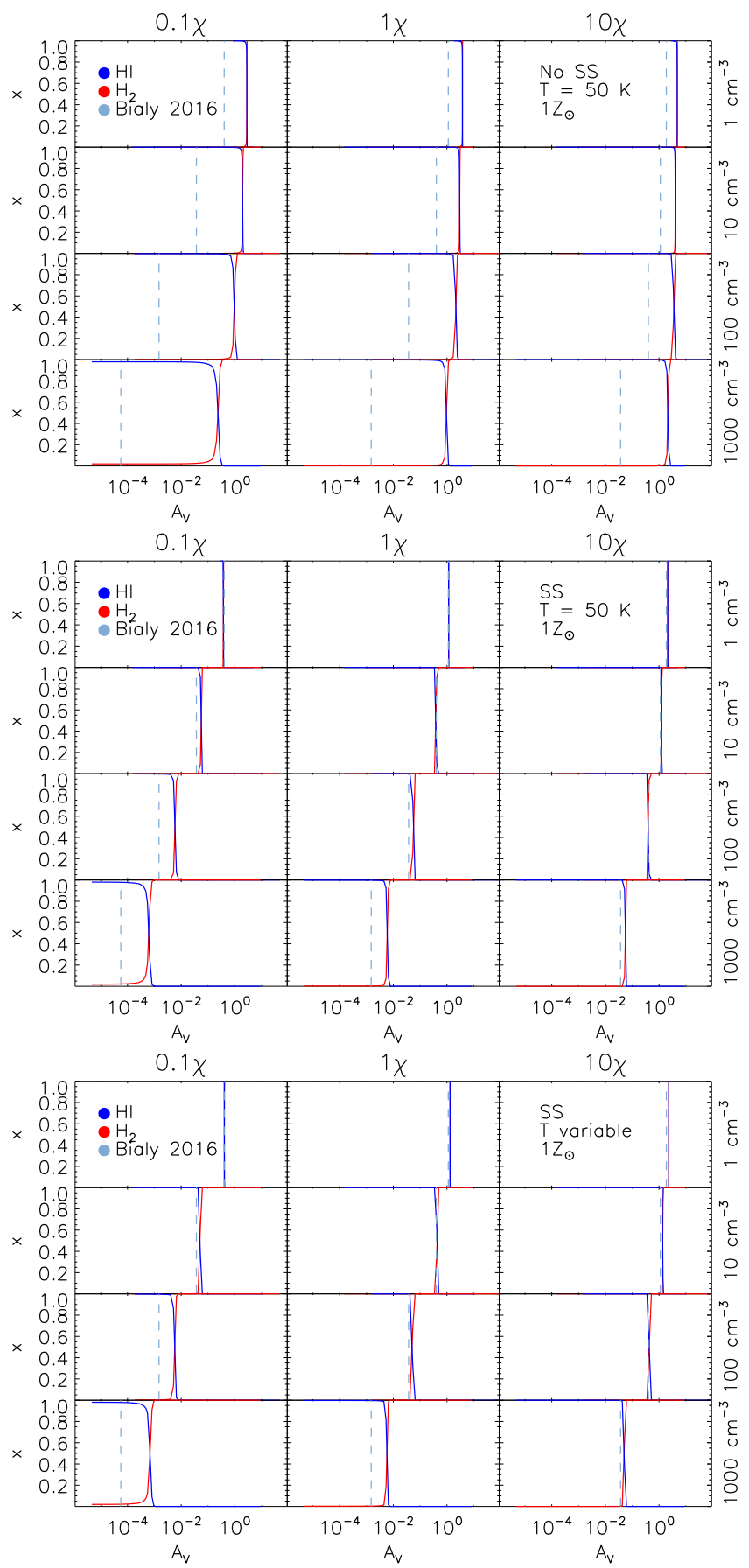

Figure 5. $\mathrm{H}$ I and $\mathrm{H}_{2}$ fraction versus visual extinction in a high density region hit by LW photons, for a range of fixed densities and fluxes, at Solar metallicity. The theoretical point of transition between $\mathrm{HI}$ and $\mathrm{H}_{2}$ is given by the the vertical dotted line calculated from Bialy \& Sternberg (2016)'s analytical function. In the top and middle plots, the temperature is fixed at $50 \mathrm{~K}$, while in the bottom plot the temperature is variable. The top plot is without self-shielding, and the bottom two plots use our self-shielding method, given by an enhancement factor of 500 for the LW photo-destruction in Equation 26
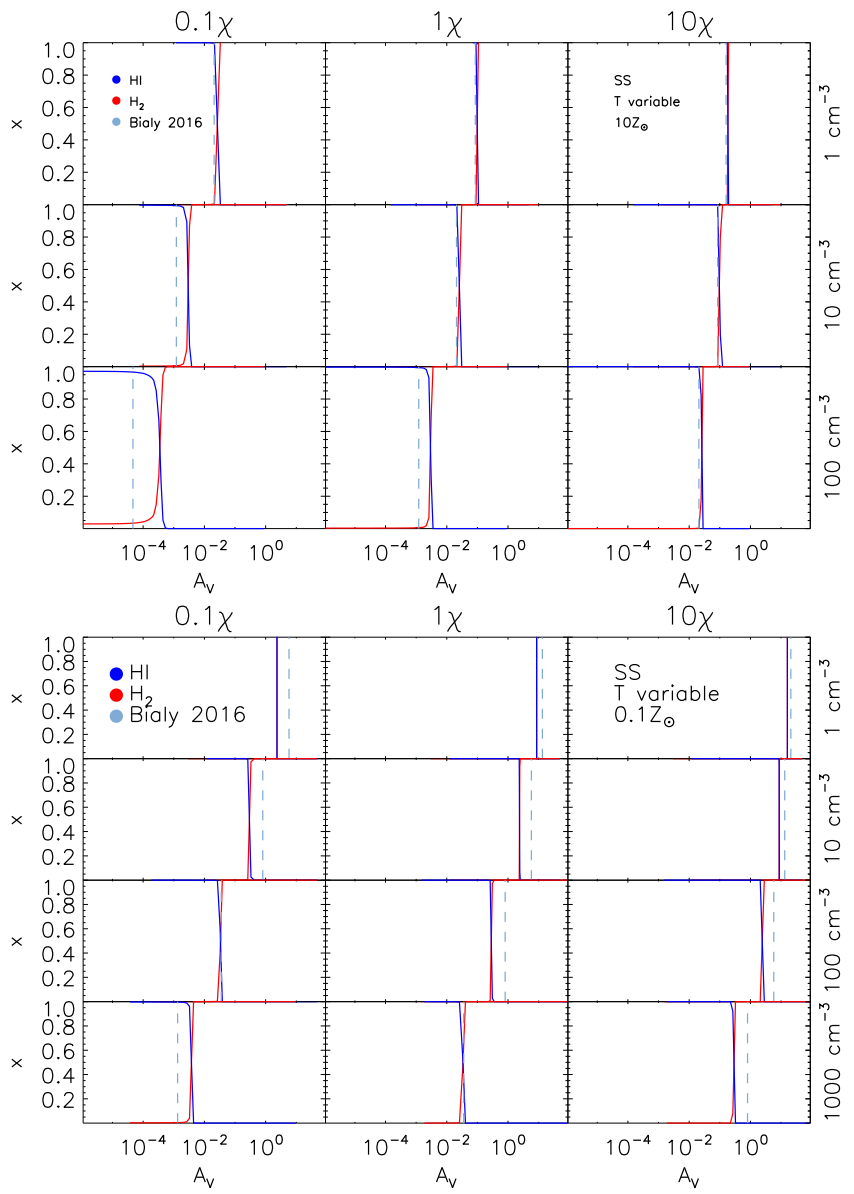

Figure 6. Same as the bottom plot in Figure 5 with self-shielding and variable temperature, but with ten times Solar metallicity (top) and a tenth of Solar metallicity (bottom).

tests, where it is dominated by our implementation of photoelectric heating (Section 2.4.2.

\subsection{PDRs in higher dimensions}

Our next step is to test our code in two- and threedimensional situations in order to verify that the results are sensible regardless of dimension, and to showcase the code's behaviour. We will radiate a circular cloud of $\mathrm{H}_{2}$ with a unidirectional LW flux, similar to the one-dimensional tests. This will show the shape of the molecular shielding region, as well as the shadow cast behind the cloud. For this scenario, using the HLL intercell flux function would be ideal, given that it is designed for beamed radiation. However, for galactic environments with isotropic sources, we will be using the GLF flux function in future work. Accordingly, we carry out these tests with the GLF function to see how the $\mathrm{H}_{2}$ dissociation behaves in multiple dimensions, and show one comparison between GLF and HLL in 3D. The HLL function simply reproduces one-dimensional test results. For a full discourse on the differences between the HLL and GLF flux functions, see Rosdahl et al. (2013).

First, in order to get a sense of the lower density regime in two dimensions we reproduce the $n=1 \mathrm{~cm}^{-3}$ row of the bottom plot in Figure 5, for $F_{L W}=0.1,1$, and $10 \chi$ and variable temperature. Instead of an infinite slab, we illuminate a 


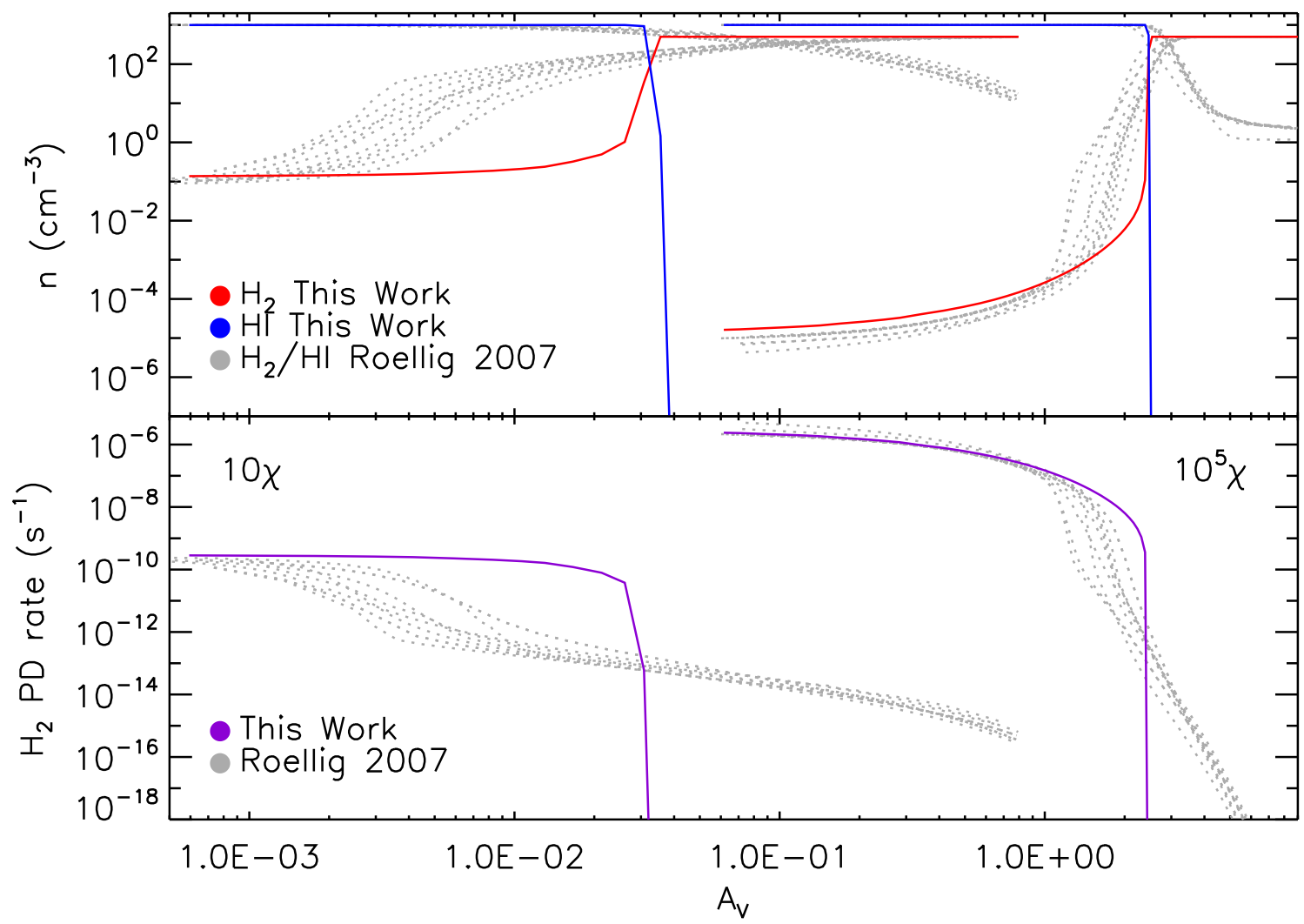

Figure 7. Hydrogen fraction number density (top) and photodissociation rate (bottom) versus visual extinction of a one-dimensional region, compared to Roellig et al. (2007)'s PDR simulations, for fixed density $10^{3} \mathrm{~cm}^{-3}$ and fluxes 10 and $10^{5} \chi$. The temperature is fixed.

higher density circle in a low density region. The box size is 3 $\mathrm{kpc}$ and the cloud radius is $0.75 \mathrm{kpc}$. The resolution is fixed at $512^{2}$ cells and we run the simulations until equilibrium is reached.

Figure 9 shows the maps of the $\mathrm{H}_{2}$ fraction, LW dissociation rate, and temperate for the circle. For each LW flux, a $\mathrm{H}_{2}$ shielding layer is apparent. There is a very thin outer layer of $\mathrm{H}$ i that protects the opaque, completely molecular core. The shape of the layer follows the geometry of the circle well. Higher values of the flux penetrate the circle more deeply and lead to higher temperatures in the shielding layer of the circle.

In each case, the flux shadow diminishes with distance from the circle because of photon crossing from the GLF function. However, despite this, there is only a small effect on the $\mathrm{H}_{2}$ content of the high density circle from dissociation by photon crossing. For the GLF flux function, the shadow is shorter lived as compared to the HLL function (Rosdahl et al. 2013), but this has little effect in a galactic context where radiation sources come from many directions.

We repeat this for the middle two rows of the bottom plot in Figure 5. where $n=10$ and $100 \mathrm{~cm}^{-3}$, and $F_{L W}=0.1,1$, and $10 \chi$. For each higher density we decrease the boxsize by a factor of ten, using $0.3 \mathrm{kpc}$ and $30 \mathrm{pc}$ respectively to preserve a well-resolved transition layer. We keep the resolution at $512^{2}$. Figures 10 and 11 show that these results are similar to the $n=1 \mathrm{~cm}^{-3}$ case, where a stronger radiation flux penetrates the circle deeper and heats it up more. The flux shadow diminishes, but this extra flux con- tinues to have a minuscule effect on the geometry of the high density $\mathrm{H}_{2}$ region in the high flux scenarios.

One matter to note is that while we increase the density by a factor of ten and decrease the boxsize by a factor of ten, the figures are not identical. As the density increases, the radiation penetrates the circle less deeply and the $\mathrm{HI}$ layer is much thinner. This highlights the non-linear nature of the problem. We leave out the bottom row of Figure 5 where $n=1000 \mathrm{~cm}^{-3}$ because we will use PDR benchmarks to test this regime.

PDR codes are overwhelmingly one-dimensional. However, two codes built for three dimensions have compared their models to the Roellig et al. (2007) benchmark. 3DPDR (Bisbas et al. 2012) takes the chemistry of UCL_PDR and ray tracing applied to a three-dimensional cloud of arbitrary density distribution. KM2 (Motoyama et al.|2015) is a hybrid hydrodynamical and chemical code. These codes are tested first in one dimension to directly compare to Roellig et al. (2007)'s tests, and test a sphere or radius $5.15 \mathrm{pc}$ hit by plane-parallel radiation, in a setup similar to benchmark test for $n=10^{3} \mathrm{~cm}^{-3}, F_{L W}=10 \chi$, and variable temperature. They find that their spheres generally agree with the benchmark from one dimension.

In order to test our model in two dimensions, we set up a circle of radius $5.15 \mathrm{pc}, n=10^{3} \mathrm{~cm}^{-3}$ and $F_{L W}=10$ and $10^{5} \chi$ with variable temperature (Figure 12). The total boxsize is $20.6 \mathrm{pc}$, with a resolution between $256^{2}$ and $8192^{2}$. Unlike the Bisbas et al. (2012) and Motoyama et al. (2015) who use ray tracing suited to PDR situations, we will continue to use the GLF to show how this function behaves. 


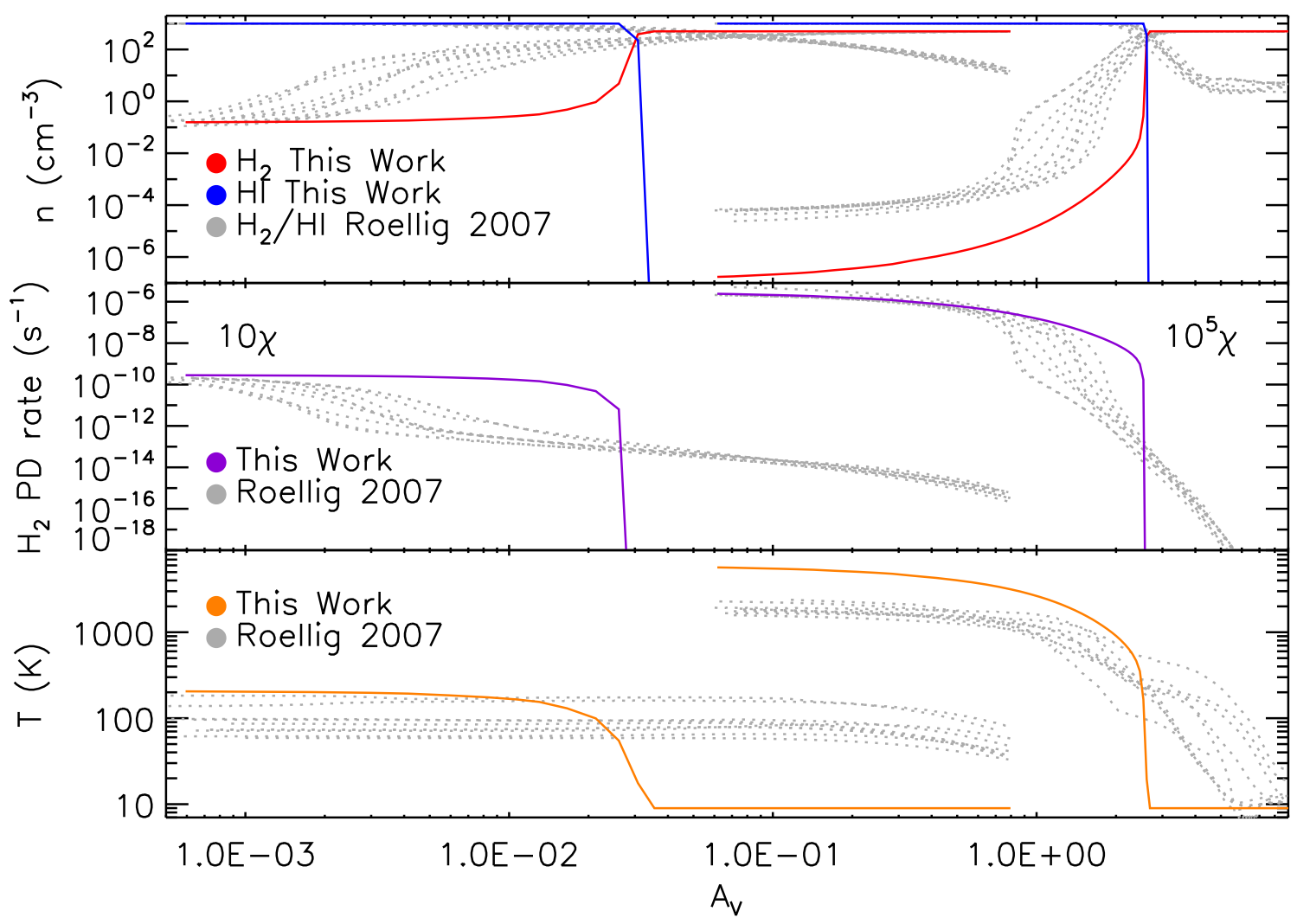

Figure 8. Hydrogen fraction number density (top), photodissociation rate (middle), and temperature (bottom) versus visual extinction of a one-dimensional region, compared to Roellig et al. (2007)'s PDR simulations, for fixed density $10^{3} \mathrm{~cm}^{-3}$ and fluxes 10 and $10^{5} \chi$. The temperature is variable.

For lower flux, our transition from atomic to molecular is shifted deeper into the circle as compared to one dimension, making it closer to the transition point for all the PDR codes. For the higher flux, the transition is also pushed deeper into the atomic region, putting it outside of the scatter of the PDR codes. However, on an absolute scale the difference is not so great. The features present in one dimension are also present in two dimensions with the abruptness of transition, and the lack of residual $\mathrm{HI}$. The shadow degradation typical to the GLF flux function is present in two dimensions, but the shape of the $\mathrm{H}_{2}$ high density region is largely unaffected.

For a three-dimensional sphere of the same setup with $F_{L W}=10^{5} \chi$ (Figure 13 and resolution fixed at $256^{3}$, we observe a similar effect where the atomic to molecular transition is slightly deeper as compared to one dimension, but matches two dimensions. This time the photon-wandering from the GLF function does lead to more noticeable $\mathrm{H}_{2}$ dissociation on the backside of the high density region. In contrast, we present contours from the same setup using the HLL function (Figure 14) suitable to beamed radiation. Here there is no shadow degradation and the dissociation of $\mathrm{H}_{2}$ remains on the beam-facing side.

The HLL and GLF flux functions both behave as expected, and our methodology for $\mathrm{H}_{2}$ chemistry remains robust in two and three dimensions.

\subsection{Strömgren spheres in a molecular medium}

A Strömgren sphere (Strömgren 1939) describes the growth of an ionisation front around a radiation source embedded in a neutral medium of hydrogen density $n_{H}$, assuming an infinite speed of light. In three dimensions, the radius of the ionisation front increases with time as,

$r_{I}(t)=r_{S \mathrm{H}_{\mathrm{I}}}\left(1-e^{-t / t_{r e c \mathrm{HI}}}\right)^{1 / 3}$,

where $r_{S H_{\text {I }}}$ is the Strömgren radius at which recombination balances ionisation and $t_{r e c H \mathrm{H}}$ is the recombination time, given by,

$r_{S \mathrm{H}_{\mathrm{I}}}=\left(\frac{3 \dot{N}_{\mathrm{H} \mathrm{I}}^{\mathrm{er}}}{4 \pi \alpha_{\mathrm{H}_{\mathrm{I}}} n_{H}^{2}}\right)^{1 / 3}$,

$t_{r e c \mathrm{H}_{\mathrm{I}}}=\left(n_{H} \alpha_{\mathrm{H} \mathrm{I}}\right)^{-1}$.

$\dot{N}_{\mathrm{H}}^{\mathrm{er}}$ is the ionising photon emission rate and $\alpha_{\mathrm{H}}$ is the recombination rate of $\mathrm{H}$ I.

Iliev et al. (2006) provides two tests for radiative transfer codes using the Strömgren sphere framework. A source of $\mathrm{H}$ I-ionising radiation emits at a rate of $\dot{N}_{\mathrm{H}}^{\mathrm{er}}=5 \times 10^{48}$ photons $\mathrm{s}^{-1}$ in a homogeneous, neutral medium of density $n_{H}=10^{-3} \mathrm{~cm}^{-3}$, with a resolution of $128^{3}$ cells. The evolution of the resulting ionisation front is then compared between codes and against the analytic solution. First the temperature is fixed at $10^{4} \mathrm{~K}$ and in the second test temperature is allowed to vary. Rosdahl et al. (2013) successfully 


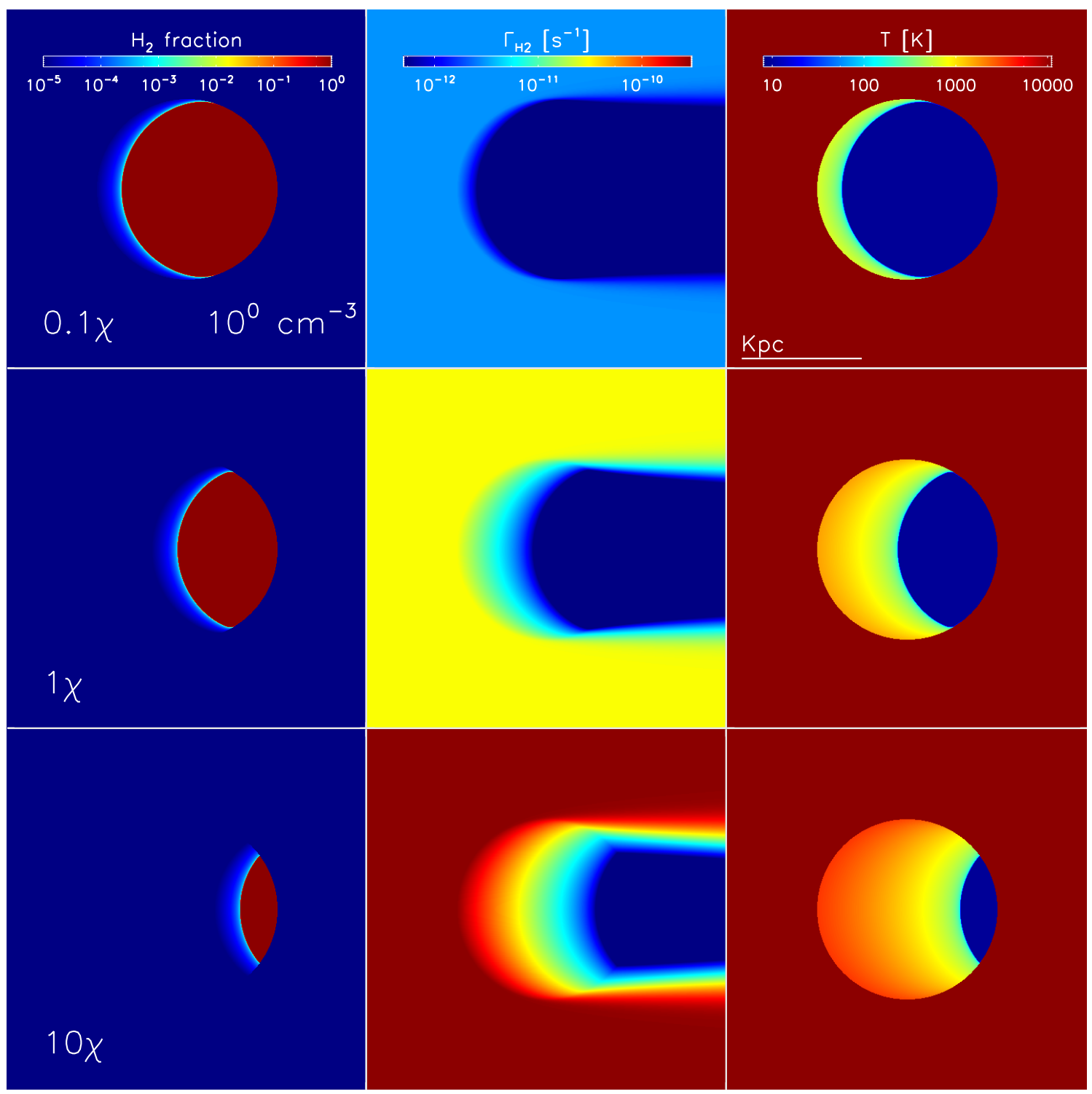

Figure 9. The hydrogen fraction number density (left), photodissociation rate (middle), and temperature (right) of a $1 \mathrm{~cm}^{-3}$ circle hit by plane-parallel LW radiation, for fluxes 0.1 (top), 1 (middle), and $10 \chi$ (bottom) using the GLF function. The temperature is variable and the boxsize $3 \mathrm{kpc}$. This is similar to the $1 \mathrm{D}$ case top in the row of the bottom plot in Figure 5

compares these two tests to both the analytic solution other codes.

We extend these tests to involve $\mathrm{H}_{2}$. Equivalent to the Strömgren sphere's Equation 45 and assuming an infinite light speed, the radius of the $\mathrm{H}_{2}$ dissociation front should grow as,

$r_{D}(t)=r_{S_{H_{2}}}\left(1-e^{-t / t_{\text {rec }} \mathrm{H}_{2}}\right)^{1 / 3}$,

where $r_{\mathrm{SH}_{2}}$ is a molecular Strömgren radius given by,

$r_{\mathrm{SH}_{2}}=\left(r_{S \mathrm{H}_{\mathrm{I}}}^{3}+\frac{3 \dot{N}_{\mathrm{H}_{2}}^{\mathrm{er}}}{4 \pi \alpha_{\mathrm{H}_{2}} n_{H}^{2}}\right)^{1 / 3}$.

$\dot{N}_{\mathrm{H}_{2}}^{\text {er }}$ is the dissociating LW photon emission rate. The formula for the $\mathrm{H}_{2}$ recombination time, $t_{\text {rec }} \mathrm{H}_{2}$, is the same as for $\mathrm{H}_{\mathrm{I}}$ (Equation 47), only with the corresponding $\alpha_{\mathrm{H}_{2}}$ as formation rate. These equations for the $\mathrm{H}_{2}$ sphere are analogous to the Hi sphere Equation 46, and we note that in
Equation 49, we add the $\mathrm{H}$ I radius to the $\mathrm{H}_{2}$ radius because the $\mathrm{H}_{2}$ sphere is expected to grow from the $\mathrm{H}$ i sphere.

For our simulations, we keep the density at $n_{H}=10^{-3}$ $\mathrm{cm}^{3}$ and begin with a fully molecular medium. The source is a supposed $4.3 \times 10^{4} \mathrm{~K} \mathrm{O}$ star with radius $10 \mathrm{R}_{\odot}$, which yields emission rates $\dot{N}_{\mathrm{H}_{2}}^{\text {er }}=3 \times 10^{48}$ and $\dot{N}_{\mathrm{H}_{\mathrm{I}}}^{\text {er }}=5 \times 10^{48}$ photons $\mathrm{s}^{-1}$. The $\mathrm{H}_{2}$ dissociation cross-section is the same as in Equation 15 and the ionisation cross-sections are averaged over a $4.3 \times 10^{4} \mathrm{~K}$ black body: $\sigma_{2 \mathrm{H}_{2}}^{N}=3.6 \times 10^{-18} \mathrm{~cm}^{2}$ and $\sigma_{2 \mathrm{H} \text { I }}^{N}=5.0 \times 10^{-18} \mathrm{~cm}^{2}$. For the fixed gas temperature test we use $3.56 \times 10^{3} \mathrm{~K}$ at which the equilibrium concentration is half molecular and half neutral, different than the initial condition. This is a little lower than the temperature for the Iliev et al. (2006) test to allow for the existence of molecular gas. We use Solar metallicity, a boxsize of $10 \mathrm{kpc}$, resolution $128^{3}$ cells, the GLF flux function, and run it for $500 \mathrm{Myr}$. For this situation set up, $r_{\mathrm{SH}_{2}}=295 \mathrm{kpc}, r_{S \mathrm{H}_{\mathrm{I}}}=4.10 \mathrm{kpc}$, 


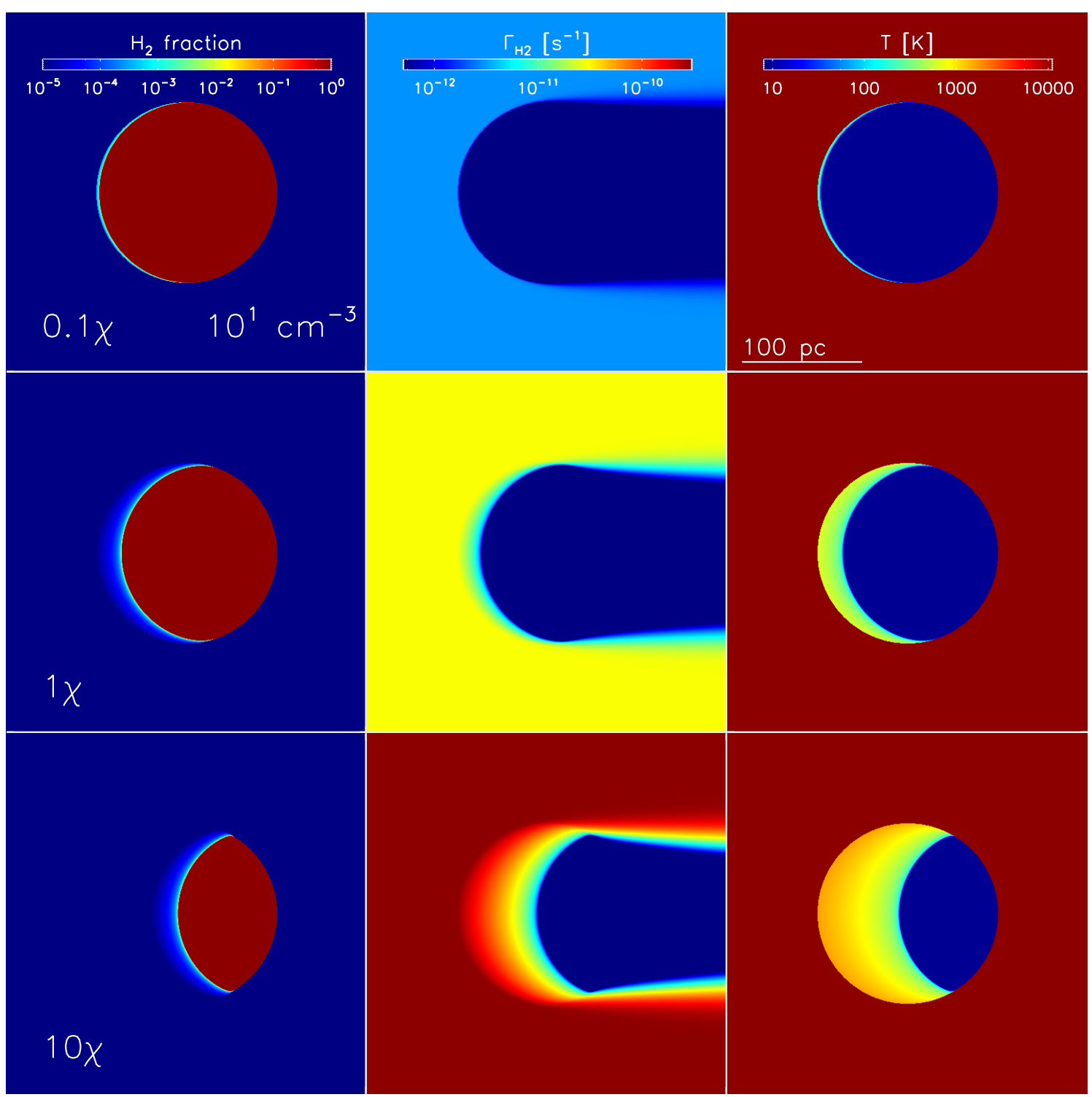

Figure 10. The hydrogen fraction number density (left), photodissociation rate (middle), and temperature (right) of a $10 \mathrm{~cm}^{-3}$ circle hit by plane-parallel LW radiation, for fluxes 0.1 (top), 1 (middle), and $10 \chi$ (bottom) using the GLF function. The temperature is variable and the boxsize 300 pc. This is similar to the $1 \mathrm{D}$ case in the second from top row of the bottom plot in Figure 5

$t_{\text {rec } \mathrm{H}_{2}}=3.33 \times 10^{7} \mathrm{Myr}$, and $t_{r e c \mathrm{H}}=53.7 \mathrm{Myr}$. For both the fixed temperature and the variable temperature tests we use two situations: without dust and $\mathrm{H}_{2}$ self-shielding, and fully shielded. The on the spot approximation (OTSA) is used, and the light speed fraction is set to $10^{-2}$ as in Rosdahl et al. (2013).

Shapiro et al. (2006) gives a relativistic expression for the $\mathrm{H}$ I-ionisation front expansion that takes a non-infinite speed of light into account:

$w=q y-\ln \left(1-y^{3}\right)$,

$w \equiv t / t_{\text {rec } \mathrm{H} \mathrm{I}}$,

$y \equiv r_{I} / r_{S H_{I}}$,

$q \equiv r_{S \mathrm{H}_{\mathrm{I}}} /\left(c_{r} t_{\text {rec }} \mathrm{H}_{\mathrm{I}}\right)$.

For a more realistic comparison of our numeric simulation to this formulation, we will use the reduced speed of light, $c_{r}$, instead of the full speed of light. Deriving an equivalent formula for the $\mathrm{H}_{2}$-dissociation front is beyond the scope of this paper.

Figure 15 gives the evolution of the $\mathrm{H}_{2}$-dissociation and $\mathrm{H}$ I-ionisation fronts without and with shielding for the fixed temperature scenario. Their evolution is compared to the analytic Equations 45 and 48 for the infinite speed of light and Equation 50 for the relativistic speed of light. Before one $\mathrm{t}_{\text {rec } \mathrm{H}}$, both the shielded and non-shielded cases grow similarly. They grow much more slowly compared to their analytic components because of the reduced speed of light, and more closely with the Shapiro et al. (2006) relativistic expression. This is also quite similar to what Rosdahl et al. (2013) found, where the analytic front is ahead of the numeric front by about $5 \%$ because the numeric front evolves more gradually than the step-wise analytic front.

Once $t_{r e c H}$ i has passed, the numeric $\mathrm{H}$ I-fronts catch up the to analytic expressions and level off at a radius of about 5 


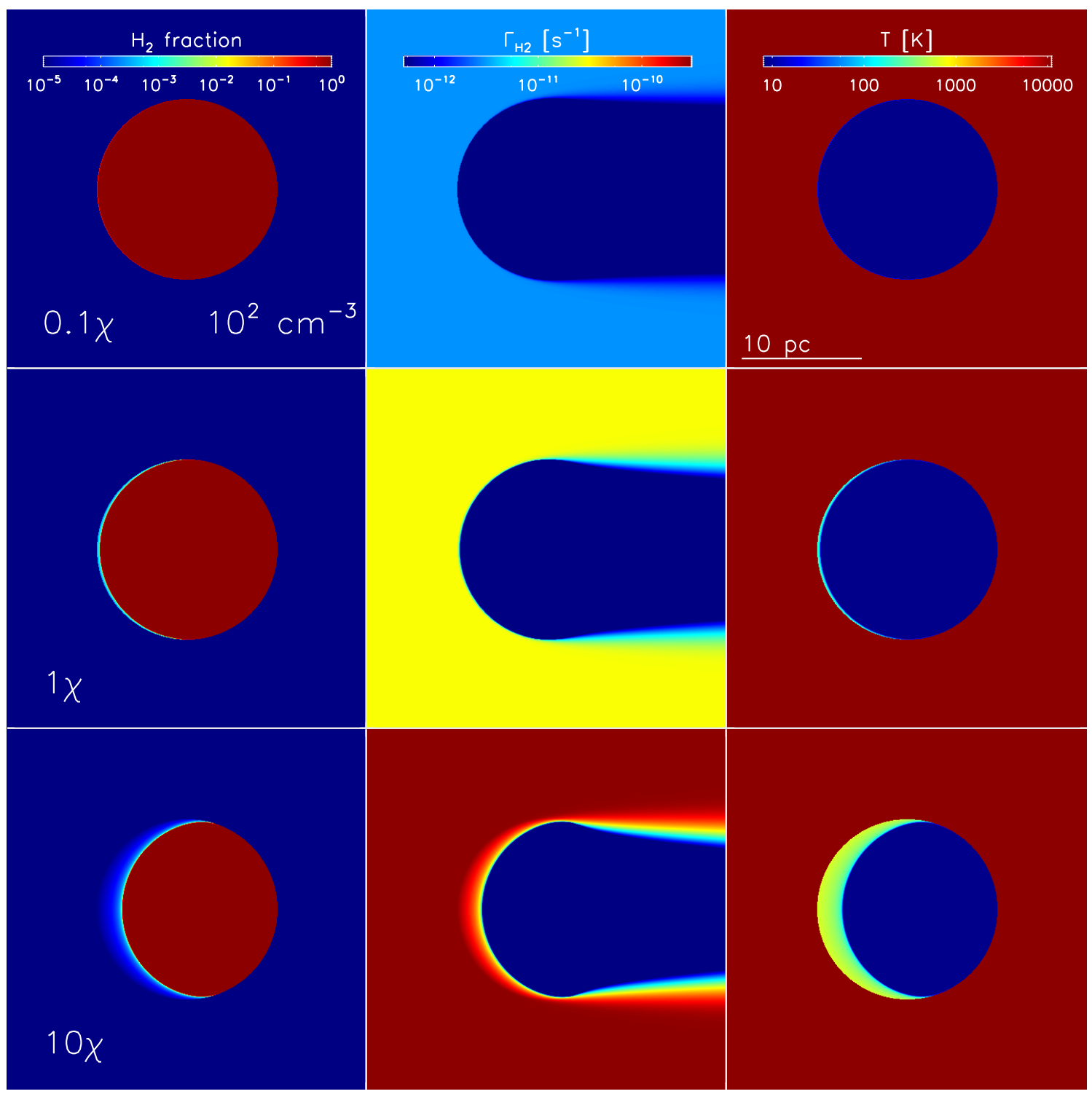

Figure 11. The hydrogen fraction number density (left), photodissociation rate (middle), and temperature (right) of a $100 \mathrm{~cm}^{-3}$ circle hit by plane-parallel LW radiation, for fluxes 0.1 (top), 1 (middle), and $10 \chi$ (bottom) using the GLF function. The temperature is variable and the boxsize $30 \mathrm{pc}$. This is similar to the $1 \mathrm{D}$ case in the second from bottom row of the bottom plot in Figure 5

kpc close to the calculated Strömgren radius of about $4 \mathrm{kpc}$. Concerning the $\mathrm{H}_{2}$-front, the unshielded and shielded cases differ after $\mathrm{t}_{\text {rec } \mathrm{H} \mathrm{I}}$. In the unshielded case, the $\mathrm{H}_{2}$-front continues to grow and reaches $8 \mathrm{kpc}$ at $500 \mathrm{Myr}$, the simulation end time. It would continue to grow, given that $t_{\text {rec }} \mathrm{H}_{2} \sim 10^{7}$ $\mathrm{Myr}$, but in reality this is much longer than the age of Universe. The shielded case, on the other hand, demonstrates the importance of both dust and $\mathrm{H}_{2}$ self-shielding. Here the $\mathrm{H}_{2}$-front levels off much like the $\mathrm{H}$ I-front, extending only slightly beyond it at around $5 \mathrm{kpc}$. This is expected because our analytic expressions do not take shielding into account.

Figure 16 shows the hydrogen fractions and radiation maps at $500 \mathrm{Myr}$, for the unshielded and shielded cases with fixed temperature. In both cases, H I stops the ionising photons and the $\mathrm{H}$ II region ends sharply. In the unshielded case, the dissociating LW photons extend much further into the
$\mathrm{H}_{2}$ layer. In the shielded case, the $\mathrm{H}_{2}$ is able to completely block the LW photons and maintain a pure molecular layer.

Next, Figure 17 gives the $\mathrm{H}_{2}$ - and $\mathrm{H}$ I-fronts for the variable temperature scenario, both unshielded and shielded. The analytic expressions from Figure 15 are left on for reference, but they are less relevant here because the variable temperature cases changes in the formation rates, and hence recombination times and Strömgren radii. With variable temperature, the growth of the fronts is similar to the

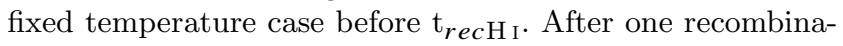
tion time, the $\mathrm{H}$ I-fronts level off to a radius slightly larger than in the fixed temperature case. Also, as in the fixed temperature case, the unshielded $\mathrm{H}_{2}$-front continues to grow towards the edge of the box, while the shielded $\mathrm{H}_{2}$-front follows the evolution of the $\mathrm{H}$ I-front at a slightly larger radius.

In Figure 18, the map of hydrogen fractions and their radiation are similar to the fixed temperature counterpoints. 

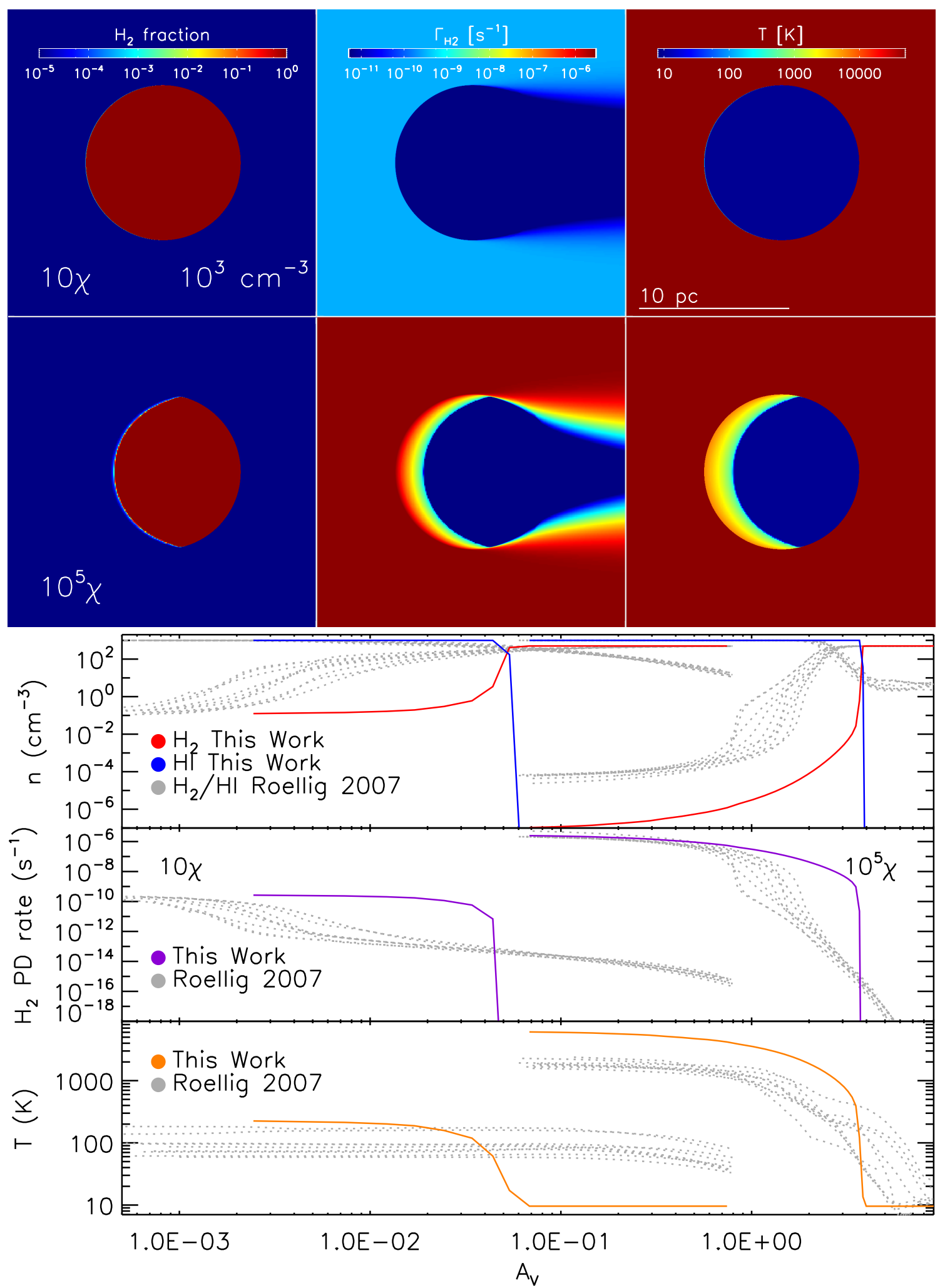

Figure 12. The hydrogen fraction number density, photodissociation rate, and temperature contour (top) and central profile (bottom) plots of a $10^{3} \mathrm{~cm}^{-3}$ circle illuminated by plane-parallel LW photons, with fluxes 10 and $10^{5} \chi$ using the GLF function. This is compared to Roellig et al. (2007)'s PDR simulations 1D. The temperature is variable and the boxsize is $20.6 \mathrm{pc}$. 

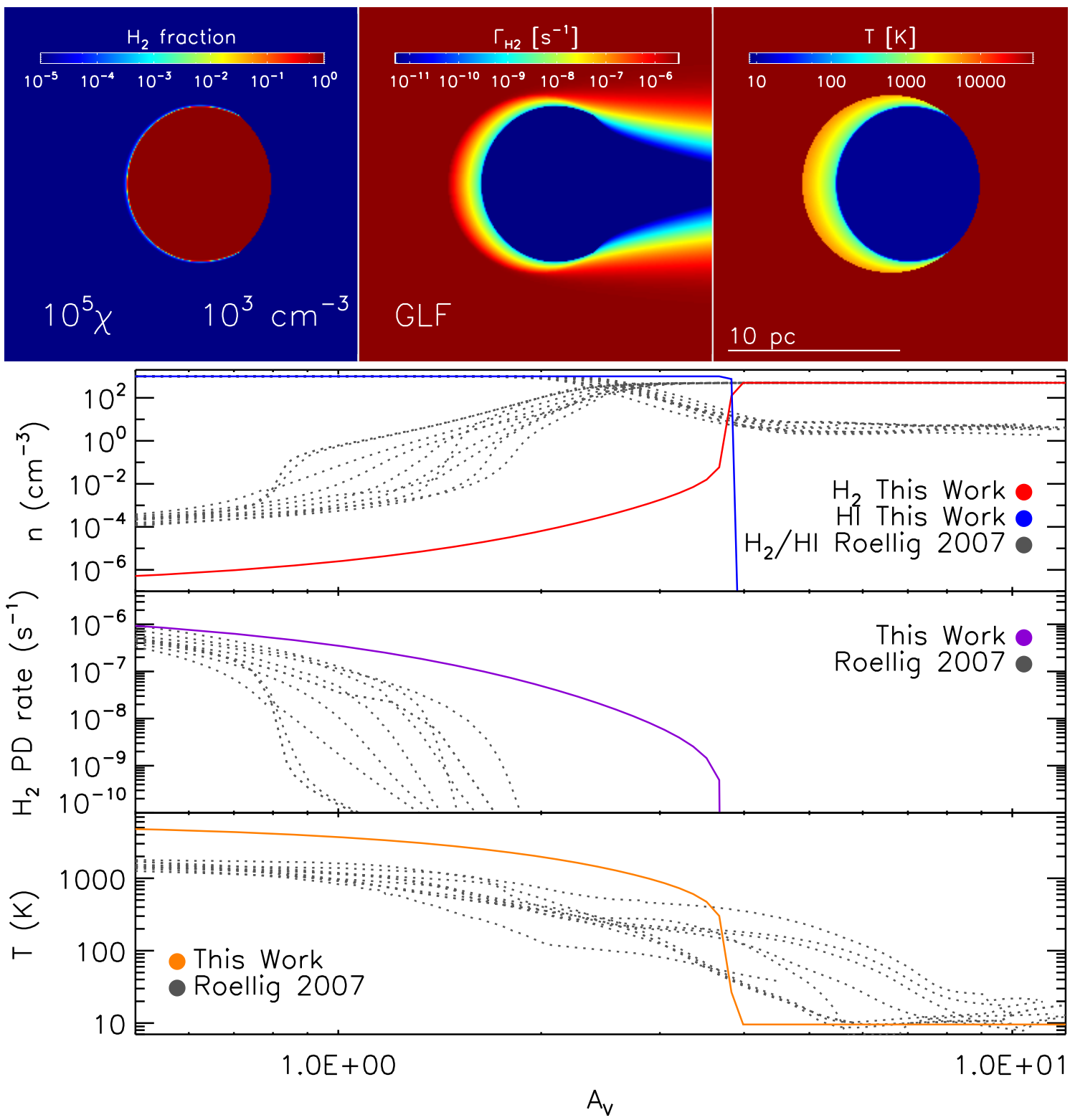

Figure 13. The hydrogen fraction, photodissociation rate, and temperature contour (top) and central profile (bottom) plots of the central slice of a $10^{3} \mathrm{~cm}^{-3}$ sphere illuminated by plane-parallel LW photons with flux $10^{5} \chi$ using the GLF function. This is compared to Roellig et al. (2007)'s PDR simulations in 1D. The temperature is variable and the boxsize is $20.6 \mathrm{pc}$.

The point of interest is in comparing the temperature maps for the unshielded and shielded cases. When $\mathrm{H}_{2}$ is shielded, the molecular region cools to the $\sim 10 \mathrm{~K}$ floor. Unshielded, the molecular region still has atomic content and cools to only $\sim 100 \mathrm{~K}$. This is reminiscent of our single cell tests (Section 3.1), where in presence of a UV background the lower density cells were unable to cool to $\sim 10 \mathrm{~K}$. Self-shielding is critical to $\mathrm{H}_{2}$ formation and molecular cooling.

Our adaptation of the Strömgren sphere to a situation involving both $\mathrm{H}_{2}$ and $\mathrm{H} \mathrm{I}$ is realistic. Our numeric results are in line with the analytic framework, and where they differ it is explained. On the shorter timescales our fronts grow more slowly than the analytic, and this is caused by the reduced speed of light for faster computation. If we do simulations where we are interested in shorter timescales then we should use the full speed of light. However, once we reach timescales of tens of Myrs and higher, our simulations grow as the analytical functions. It is theses longer timescales that are of interest to our galactic application of this methodology.

\section{SUMMARY AND FUTURE}

In this paper we present our molecular addition to RAMSESRT, an AMR hydro-dynamical code with radiative transfer. We follow the non-equilibrium evolution of molecular, atomic, and ionised hydrogen coupled to the radiative transfer of the dissociating Lyman-Werner and ionising photons. Our moment-based radiative transfer uses the Eddington tensor approximation for closure. Because this method is purely local, we gain tremendously in computational time independent of the number of sources. A semi-implicit method 


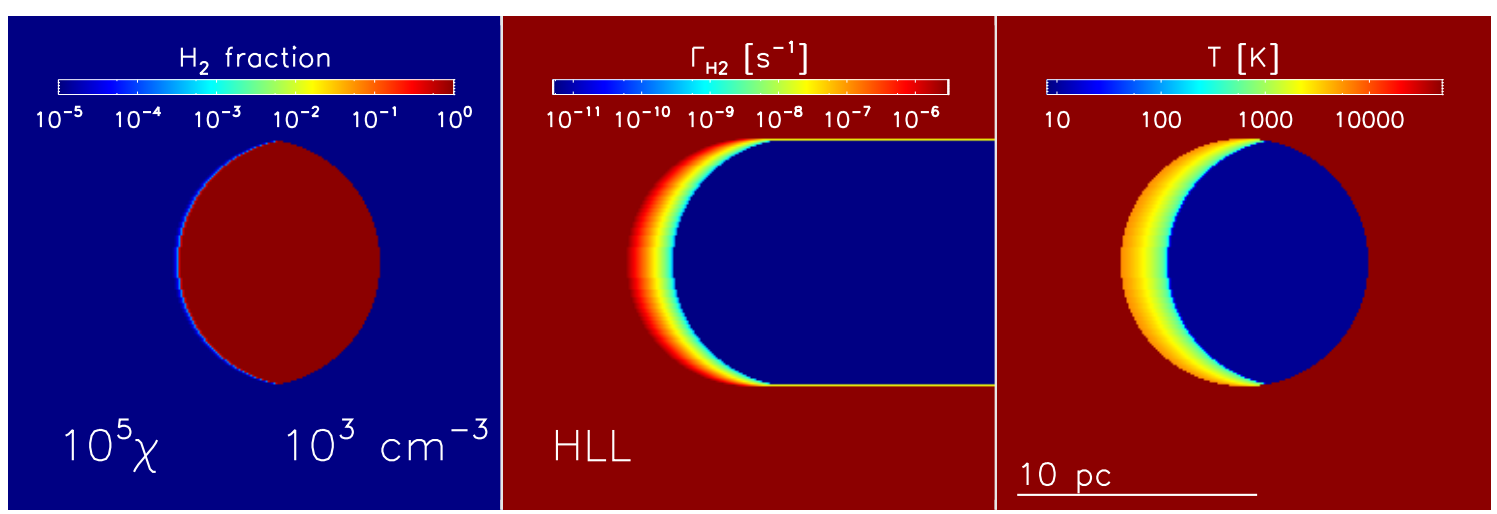

Figure 14. The hydrogen fraction, photodissociation rate, and temperature contour plots of the central slice of a $10^{3} \mathrm{~cm}^{-3}$ sphere illuminated by plane-parallel LW photons with flux $10^{5} \chi$ using the HLL function. This is compared to Roellig et al. (2007)'s PDR simulations in $1 \mathrm{D}$. The temperature is variable and the boxsize is $20.6 \mathrm{pc}$.

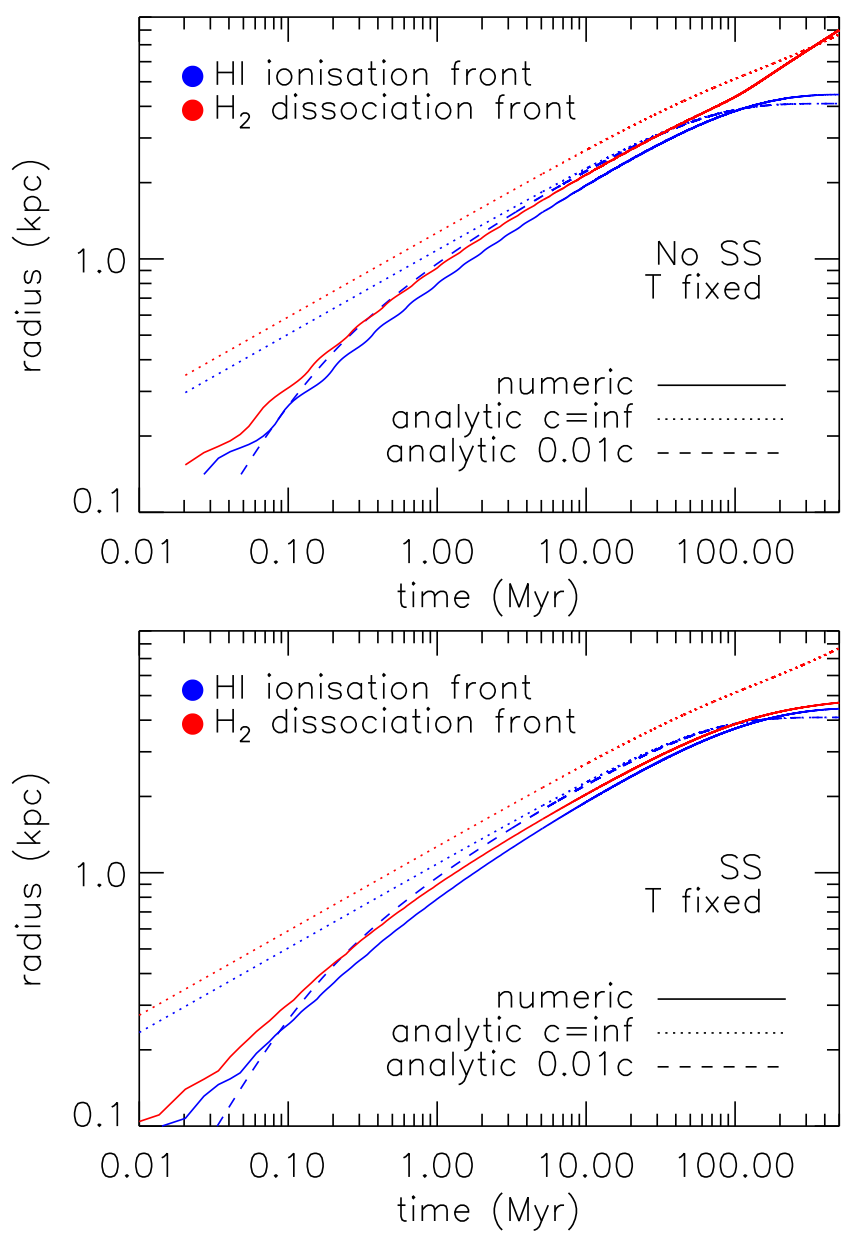

Figure 15. The evolution of $\mathrm{H}_{\mathrm{I}}$ ionisation and $\mathrm{H}_{2}$ dissociation fronts up to $500 \mathrm{Myr}$ for a Strömgren-like scenario with fixed temperature and $10^{-3} \mathrm{~cm}^{-3}$. Top: without dust and self-shielding of $\mathrm{H}_{2}$. Bottom: with $\mathrm{H}_{2}$ and dust. The boxsize is $10 \mathrm{kpc}$. Solid lines follow our simulations. The dotted lines follow Equations 45 and 48 The dashed line follows the reduced speed of light equation given in Shapiro et al. (2006). advances our thermal chemistry rate equations in time, and species fractional abundances are fully coupled to temperature, radiation, and hydrodynamics. The chemical processes we include for $\mathrm{H}$ I are recombination, destruction by electron collision, and photoionisation; for $\mathrm{H}_{2}$ we include formation catalysed by dust grains and primordial gas phase formation in the absence of metals, collisional destruction with atomic hydrogen and itself, and photo-destruction by dissociating LW photons and higher energy ionising photons.

We capitalise on our moment-based radiative transfer to introduce a new method of modelling $\mathrm{H}_{2}$ self-shielding against LW photons. We boost the destruction of LW photons that dissociate $\mathrm{H}_{2}$ by a constant factor to incorporate the fact that only a fraction of LW photon absorption leads to $\mathrm{H}_{2}$ dissociation. As the $\mathrm{LW}$ photons continue to travel through gas cells rich in $\mathrm{H}_{2}$ across many timesteps, their repeated destruction mimics a column density. This differs from works by other authors where $\mathrm{H}_{2}$ self-shielding is implemented by converting a volume density to a column density and decreasing $\mathrm{H}_{2}$ destruction.

A suite of tests demonstrate the robustness of our method across an array of situations.

Single cells: Our single cell tests evolve the hydrogen chemistry in zero dimensions, for a grid of initial temperatures, fixed densities, and initial atomic/ionised fractions. The four scenarios are: fixed or variable temperatures, and with or without a UV background flux. In the fixed temperature cases, the cells evolve to the expected equilibrium states given enough time. Around $10^{4} \mathrm{~K}$ the final state is entirely atomic, while higher temperatures are fully ionised and lower fully molecular. With a UV background, the final state is also dependent on density and higher density cells give increasingly molecular final states. With evolving temperature, the cells cool down to the expected $\sim 10 \mathrm{~K}$ floor. Cooling occurs faster with increasing density and decreasing initial ionisation fraction. In the presence of a UV background, lower density cells are unable to cool down to this floor, and the final temperature is dependent on cell density.

Self-shielding calibration: We calibrate our selfshielding model with one-dimensional simulations. A constant flux of LW photons hits a high density $\mathrm{H}_{2}$ region, and we repeat this for a grid of constant densities and LW fluxes. In each high-density region, the photons dissociate the $\mathrm{H}_{2}$ 
Sarah Nickerson et al.

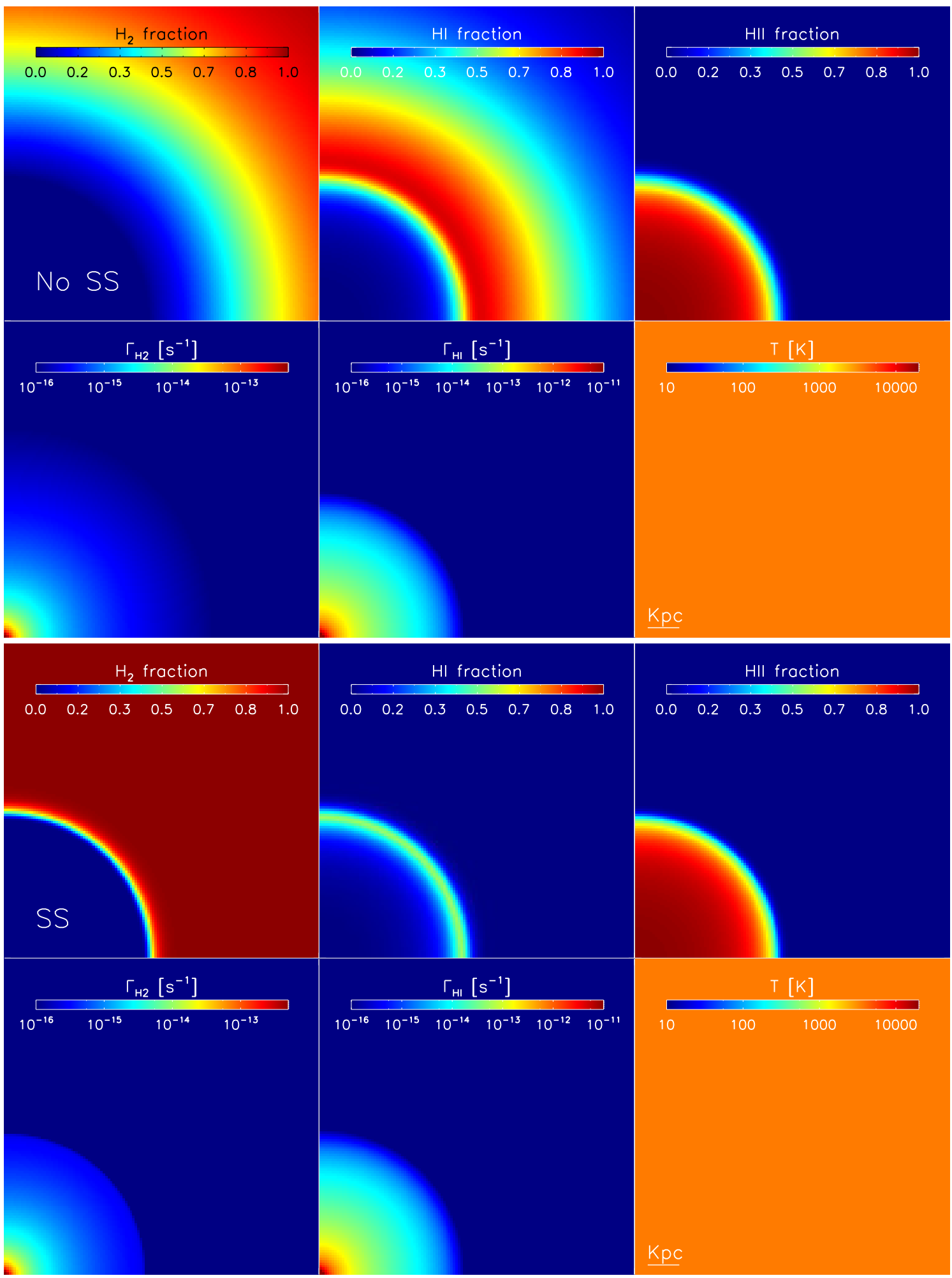

Figure 16. Central slices for our Strömgren-like scenario at $500 \mathrm{Myr}$, with fixed temperature and boxsize 10 kpc. Contours are given for $\mathrm{H}_{2}, \mathrm{HI}$, and $\mathrm{H}$ II fractions, $\mathrm{H}_{2}$ and $\mathrm{H}$ I photodissociation rates, and temperature. Top two rows: unshielded case. Bottom two rows: shielded case. 


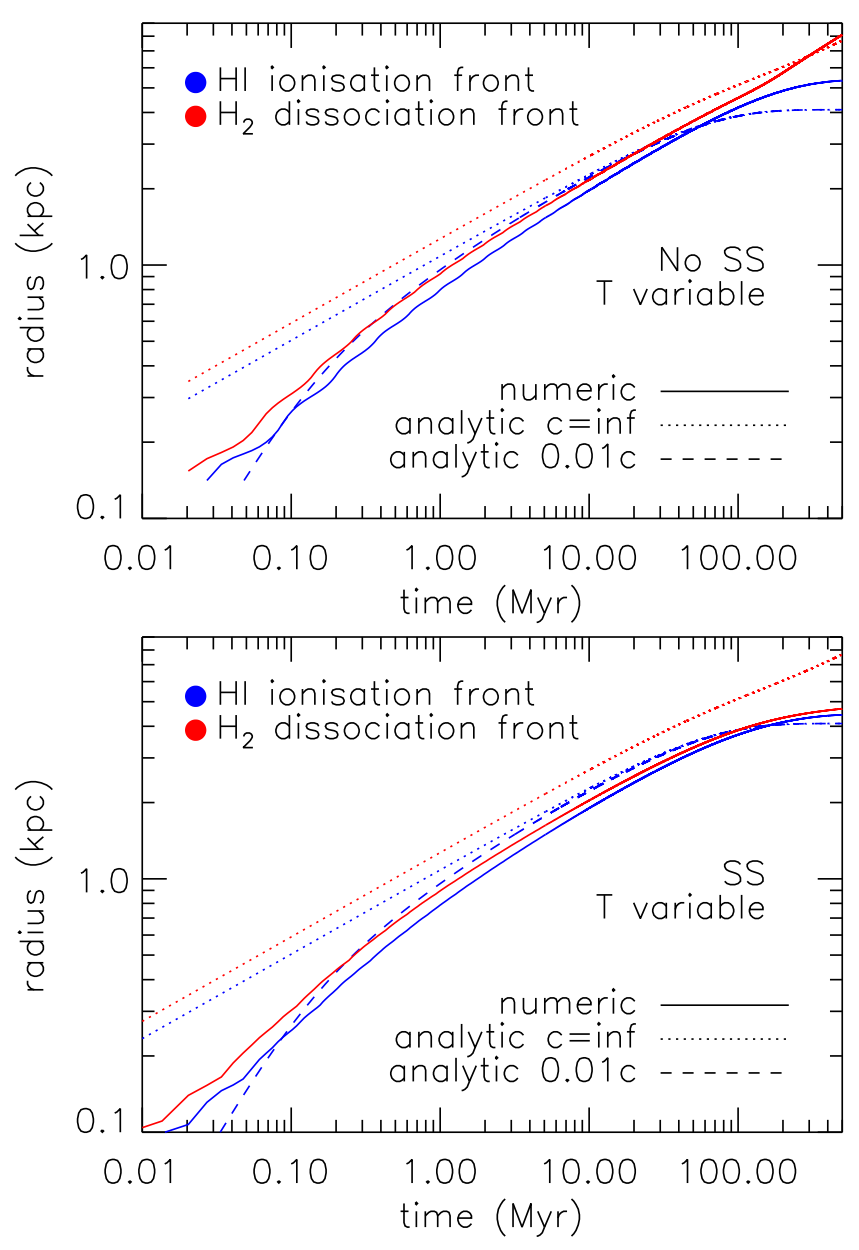

Figure 17. The evolution of $\mathrm{H}_{\mathrm{I}}$ ionisation and $\mathrm{H}_{2}$ dissociation fronts up to 500 Myr for a Strömgren-like scenario with variable temperature and density $10^{-3} \mathrm{~cm}^{-3}$. Top: without dust and selfshielding of $\mathrm{H}_{2}$. Bottom: with $\mathrm{H}_{2}$ and dust. The boxsize is 10 kpc. Solid lines follow our simulations. The dotted lines follow Equations 45 and 48 for fixed $T=3.56 \times 10^{3} \mathrm{~K}$. The dashed line follows the reduced speed of light equation given in Shapiro et al. (2006) for fixed $T=3.56 \times 10^{3} \mathrm{~K}$.

into H I until the photons are all destroyed by dissociation, leaving an $\mathrm{H}_{2}$ core. We compare our $\mathrm{H}$ I- $\mathrm{H}_{2}$ transition depth to the analytic prediction by Bialy \& Sternberg (2016), and without self-shielding the photons penetrate the $\mathrm{H}_{2}$ region too deeply. We experiment with constants by which to boost the LW photo-destruction, and found one factor that reproduces the analytic results satisfactorily for each flux, density, and metallicity. Our method works for both fixed and variable temperatures scenarios.

PDR code comparison: We compare the results from our code to the one-dimensional Roellig et al. (2007) benchmark tests, which comprises of ten separate PDR codes. For a high density region of $10^{3} \mathrm{~cm}^{-3}$, the four scenarios we test are fluxes 10 or $10^{5} \chi$, and the temperature constant at 50 $\mathrm{K}$ or variable. Our transition depth between $\mathrm{H}_{2}$ and $\mathrm{HI}_{\mathrm{I}}$ is accurate. However, we are unable to reproduce the exact PDR transition shape. The photodissociation rate and temperature profiles follow a similar exponential trend. This is because the one-dimensional PDR codes are able to use a column density-based power law for their $\mathrm{H}_{2}$ self-shielding, while we use a local exponential form. Because of this, the small-scale physics of our transition region are inexact. However, because we will be applying the code to large scale simulations the PDR curve is unimportant to us. The important quantity to reproduce is a transition zone, which we do successfully.

Higher dimensions: We illuminate a high density, circular $\mathrm{H}_{2}$ region in a low density region with plane-parallel LW radiation for a range of fixed densities and fluxes following the GLF function, with variable temperature. The molecular dissociation region of the circle follows a smooth, symmetric shape. The flux shadow tapers according to the GLF function, but this does not appreciably affect the molecular abundance or the temperature of the high density region. In two dimensions, the results match the expectation from comparable one-dimensional simulations, which we draw from our self-shielding calibration tests and the Roellig et al. (2007) benchmarks. We also illuminate a high density, spherical $\mathrm{H}_{2}$ region and it performs to expectations, aside from some leaked photons as expected from the GLF flux function, while the HLL function produces a crisp shadow as anticipated.

Strömgren Sphere: The Strömgren sphere models the growth of an OB star's ionisation front. Traditionally, this test is done in a neutral medium but we expand it to a molecular medium. Analytical expressions predict the growth of these ionisation and dissociation fronts. We compare our numeric results to the analytical expressions for four scenarios: with temperature fixed and variable, and with and without self-shielding. An H II sphere encapsulates the source, while an $\mathrm{H}$ i shell separates it from the outer $\mathrm{H}_{2}$ medium. The $\mathrm{H}$ Ifront grows in line with expectations for numeric Strömgren spheres. Our $\mathrm{H}_{2}$-front grows at a similar speed as the $\mathrm{HI}$ front up until the $\mathrm{H}$ Irecombination time. After this, the the presence of self-shielding determines the $\mathrm{H}_{2}$-front's growth. Without self-shielding the $\mathrm{H}_{2}$-front continues to grow, while with self-shielding the growth slows in step with the $\mathrm{H}$ Ifront. When we vary the temperature, the molecular region cools to our $\sim 10 \mathrm{~K}$ floor with self-shielding, while without self-shielding the gas is unable to cool so low.

The importance of $\mathrm{H}_{2}$ self-shielding is manifest in our simulations. Without it, deep $\mathrm{H}_{2}$ cores cannot form and the gas is unable to cool because $\mathrm{H}_{2}$ is a critical coolant of interstellar gas. Our self-shielding implementation uses entirely local methods, and distills the complex physics involved into a computationally expedient format optimized for large scale galaxy simulations. In this paper we only model fixed density situations with the hydrodynamics turned off. Our future work will be to run galaxies with the full suite of hydrogen and helium chemistry, radiative transfer, and hydrodynamics.

There are several outstanding questions concerning the hydrogen content of observed galaxies that the molecular addition to RAMSES-RT is uniquely poised to answer. Traditionally the $\mathrm{H}_{2}$ content of galaxies is calculated from a conversion factor between the easily-observable carbon monoxide $(\mathrm{CO})$ and the observationally-obtuse $\mathrm{H}_{2}$. However, a growing body of evidence suggests a CO-dark components to the molecular ISM (Tielens \& Hollenbach 1985. Wolfire et al. 2010 Smith et al. 2014). We can explore this conversion factor by adding $\mathrm{CO}$ chemistry analogous to $\mathrm{H}_{2}$ into RAMSES-RT . The origins of H I high velocity clouds (HVCs) 


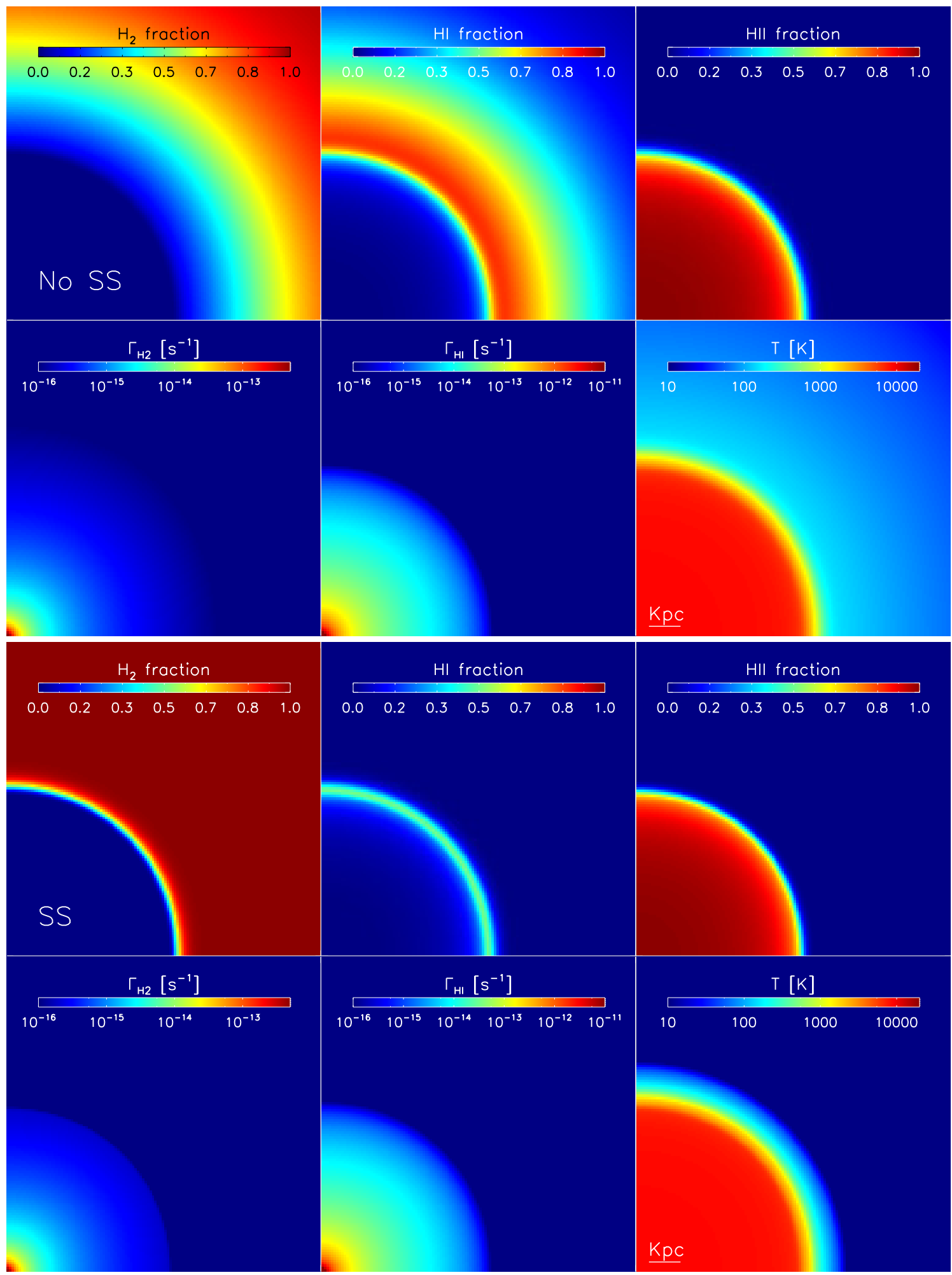

Figure 18. Central slices for our Strömgren-like scenario at $500 \mathrm{Myr}$, with variable temperature and boxsize 10 kpc. Contours are given for $\mathrm{H}_{2}, \mathrm{HI}$, and $\mathrm{H}$ II fractions, $\mathrm{H}_{2}$ and $\mathrm{H}_{\text {I }}$ photodissociation rates, and temperature. Top two rows: unshielded case. Bottom two rows: shielded case. 
outside our galaxy and others remains a mystery (Muller et al. 1963 Wakker \& van Woerden 1997, Wakker 2001). Because we now fully characterize the $\mathrm{H}$ I content in our model, we will be able to identify HVCs and track their origin. Furthermore, we can bring our chemistry model to a cosmological context. This realm hosts the "too big to fail" problem (Boylan-Kolchin et al. 2011) where $\Lambda$ CDM simulations predict subhalos that are too dense to host any measured satellites from matching observed galaxies (Papastergis et al. 2014). The ALFALFA survey (Haynes et al. 2011) infers the size of such galaxies via $\mathrm{H}$ I measurements, and our model can be a useful tool to connect these observations to cosmological galaxy simulations. By modelling the $\mathrm{H}_{2}$ chemistry on a cell-by-cell basis, we build a foundation on which to explore even the largest of galactic problems.

\section{ACKNOWLEDGEMENTS}

SN is supported by the UZH Candoc Scholarship, and performed these simulations on the Piz Daint supercomputer in CSCS Lugano. JR was funded by the European Research Council under the European Union's Seventh Framework Programme (FP7/2007-2013) / ERC Grant agreement 278594-GasAroundGalaxies and the ORAGE project from the Agence Nationale de la Recherche under grant ANR-14CE33-0016-03.

\section{REFERENCES}

Abel T., Anninos P., Zhang Y., Norman M. L., 1997, New Astronomy, 2, 181

Abgrall H., Le Bourlot J., Pineau G., Roueff E., Flower D. R., Heck L., 1992, Astronomy \& Astrophysics, 253, 525

Anninos P., Zhang Y., Abel T., Norman M. L., 1997, New Astronomy, 2, 209

Aubert D., Teyssier R., 2008, Monthly Notices of the Royal Astronomical Society 387, 295

Bialy S., Sternberg A., 2016, Astrophysical Journal p. 13

Bigiel F., Leroy A., Walter F., Brinks E., de Blok W. J. G., Madore B., Thornley M. D., 2008, The Astronomical Journal 136, 2846

Bisbas T. G., Bell T. A., Viti S., Yates J., Barlow M. J., 2012, Monthly Notices of the Royal Astronomical Society, 427, 2100

Black J. H., van Dishoeck E. F., 1987, The Astrophysical Journal, 322,412

Bolatto A. D., Wolfire M., Leroy A. K., 2013, Annual Review of Astronomy and Astrophysics 51, 207

Boylan-Kolchin M., Bullock J. S., Kaplinghat M., 2011, Monthly Notices of the Royal Astronomical Society: Letters, 415, 40

Butler M. J., Tan J. C., Teyssier R., Rosdahl J., Van Loo S., Nickerson S., 2017, The Astrophysical Journal 841, 82

Capelo P. R., Bovino S., Lupi A., Schleicher D. R. G., Grassi T., 2017, Monthly Notices of the Royal Astronomical Society, 000

Christensen C., Quinn T., Governato F., Stilp A., Shen S., Wadsley J., 2012, Monthly Notices of the Royal Astronomical Society. 425,3058

Dove J. E., Mandy M. E., 1986, The Astrophysical Journal 311, L93

Draine B. T., 1978, The Astrophysical Journal Supplement Series, 36,595

Draine B. T., Bertoldi F., 1996, The Astrophysical Journal 468, 50
Ferland G. J., Korista K. T., Verner D. A., Ferguson J. W., Kingdon J. B., Verner E. M., 1998, Publications of the Astronomical Society of the Pacific 110, 761

Forbes J. C., Krumholz M. R., Goldbaum N. J., Dekel A., 2016, Nature Publishing Group 535, 523

Fu J., Guo Q., Kauffmann G., Krumholz M. R., 2010, Monthly Notices of the Royal Astronomical Society, 409, 515

Galli D., Palla F., 1998, Astronomy \& Astrophysics 420, 19

Glover S. C. O., Abel T., 2008, Monthly Notices of the Royal Astronomical Society, 388, 1627

Glover S. C. O., Mac Low M. M., 2007, The Astrophysical Journal 169, 239

Gnedin N. Y., Abel T., 2001, New Astronomy 6, 437

Gnedin N. Y., Draine B. T., 2014, The Astrophysical Journal 795,37

Gnedin N. Y., Kravtsov A. V., 2011, The Astrophysical Journal 728,88

Gnedin N. Y., Tassis K., Kravtsov A. V., 2009, The Astrophysical Journal, 697, 55

Gould R. J., Salpeter E. E., 1963, The Astrophysical Journal, 138, 393

Guhathakurta P., Draine B. T., 1989, The Astrophysical Journal 345,230

Haardt F., Madau P., 1996, The Astrophysical Journal 461, 20

Habing H. J., 1968, Bull. Astr. Inst. Netherlans, 19, 421

Haiman Z., Abel T., Rees M. J., 2000, The Astrophysical Journal 534,11

Halle A., Combes F., 2013, Astronomy \& Astrophysics 559, A55

Harten A., Lax P. D., van Leer B., 1983, SIAM Review, 25, 35

Haynes M. P., et al., 2011, The Astronomical Journal, 142, 170

Hollenbach D., McKee C. F., 1979, The Astrophysical Journal Supplement Series 41, 555

Hollenbach D., Tielens A., 1999, Reviews of Modern Physics 71, 173

Hopkins P. F., Kereš D., Oñorbe J., Faucher-Giguère C. A., Quataert E., Murray N., Bullock J. S., 2014, Monthly Notices of the Royal Astronomical Society, 445, 581

Hu C.-y., Naab T., Walch S., Glover S. C. O., Clark P. C., 2016, Monthly Notices of the Royal Astronomical Society, 3553, 3528

Iliev I. T., et al., 2006, Monthly Notices of the Royal Astronomical Society 371, 1057

Iliev I. T., et al., 2009, Monthly Notices of the Royal Astronomical Society, 400, 1283

Kamp I., Bertoldi F., 2000, Astronomy \& Astrophysics, 353, 276

Katz H., Kimm T., Sijacki D., Haehnelt M., 2017, Monthly Notices of the Royal Astronomical Society, 468, 4831

Kennicutt R. C., 1998, The Astrophysical Journal, 498, 541

Kuhlen M., Krumholz M. R., Madau P., Smith B. D., Wise J., 2012, The Astrophysical Journal 36

Larson R. B., 1981, Monthly Notices of the Royal Astronomical Society 194, 809

Lax P. D., 1954, Communications on Pure and Applied Mathematics, 7, 159

Le Bourlot J., Pineau Des Forets G., Roueff E., Flower D. R., 1993, Astronomy \& Astrophysics, 267, 233

Lee H.-H., Herbst E., Pineau des Forets G., Roueff E., Le Bourlot J., 1996, Astronomy \& Astrophysics, 311, 690

Leroy A. K., et al., 2013, The Astronomical Journal, 146, 19

Levermore C. D., 1984, Journal of Quantitative Spectroscopy and Radiative Transfer 31,149

Lupi A., Bovino S., Capelo P. R., Volonteri M., Silk J., 2017, Monthly Notices of the Royal Astronomical Society, 000, 1

Martin P. G., Keogh W. J., Mandy M. E., 1998, The Astrophysical Journal, 499, 793

McKee C. F., Krumholz M. R., 2010, The Astrophysical Journal 709,308

McKee C. F., Ostriker E. C., 2007, Annual Review of Astronomy 
and Astrophysics, 45, 565

Meijerink R., Spaans M., 2005, Astronomy \& Astrophysics 436, 397

Mihalas D., Mihalas B. W., 1984, Foundations of radiation hydrodynamics. New York, Oxford University Press

Motoyama K., Morata O., Hsien S., Ruben K., Hasegawa T., 2015, The Astrophysical Journal 808

Muller C., Oort J., Raimond E., 1963, Academie des Sciences Paris Comptes Rendus, 257, 1661

Pallottini A., Ferrara A., Bovino S., Vallini L., Gallerani S., Maiolino R., Salvadori S., 2017, Monthly Notices of the Royal Astronomical Society 18, 1

Papastergis E., Giovanelli R., Haynes M. P., Shankar F., 2014, Astronomy \& Astrophysics, 113, 15

Pelupessy F. I., Papadopoulos P. P., Van Der Werf P., 2006, The Astrophysical Journal, 645, 1024

Richings A. J., Schaye J., 2016, Monthly Notices of the Royal Astronomical Society 458, 270

Robertson B. E., Kravtsov A. V., 2008, The Astrophysical Journal 680,1083

Roellig M., et al., 2007, Astronomy \& Astrophysics, p. 28

Rosdahl J., Teyssier R., 2015, Monthly Notices of the Royal Astronomical Society 449, 4380

Rosdahl J., Blaizot J., Aubert D., Stranex T., Teyssier R., 2013, Monthly Notices of the Royal Astronomical Society, 436, 2188

Rosdahl J., Schaye J., Dubois Y., Kimm T., Teyssier R., 2017, Monthly Notices of the Royal Astronomical Society, 466, 11

Rosen A., Bergman J. N., 1995, The Astrophysical Journal, 440, 634

Schmidt M., 1959, The Astrophysical Journal, 129, 243

Schruba A., et al., 2011, The Astronomical Journal 142, 37

Shapiro P. R., Iliev I. T., Alvarez M. A., Scannapieco E., 2006, Astrophysical Journal Letters v.489 648, 922

Smith R. J., Glover S. C. O., Clark P. C., Klessen R. S., Springel V., 2014, MNRAS 441, 1628

Solomon P. M., Rivolo a. R., Barrett J., Yahil A., 1987, The Astrophysical Journal, 319, 730

Somerville R. S., Popping G., Trager S. C., 2015, Monthly Notices of the Royal Astronomical Society, 453, 4337

Stecher T. P., Williams D. A., 1967, Astrophysical Journal Letters 149,29

Sternberg A., Dalgarno A., 1989, The Astrophysical Journal, 338, 197

Sternberg A., Le Petit F., Roueff E., Le Bourlot J., 2014, The Astrophysical Journal, 790, 10

Stoerzer H., Stutzki J., Sternberg A., 1996, Astronomy \& Astrophysics, 310, 592

Strömgren B., 1939, The Astrophysical Journal, 89, 526

Taylor S. D., Hartquist T. W., Williams D. A., 1993, Monthly Notices of the Royal Astronomical Society, 264, 929

Teyssier R., 2002, Astronomy \& Astrophysics 385, 337

Thompson R., Nagamine K., Jaacks J., Choi J.-H., 2014, The Astrophysical Journal, 780, 145

Tielens A. G. G. M., Hollenbach D., 1985, The Astrophysical Journal, 291, 722

Tomassetti M., Porciani C., Romano-Diaz E., Ludlow A. D., 2014, Monthly Notices of the Royal Astronomical Society, 446, 3330

Wakker B. P., 2001, The Astrophysical Journal Supplement Series 136, 463

Wakker B. P., van Woerden H., 1997, Annual Review of Astronomy and Astrophysics, 35, 217

Williams J. P., McKee C. F., 1997, The Astrophysical Journal, 476, 166

Wolcott-Green J., Haiman Z., Bryan G. L., 2011, Monthly Notices of the Royal Astronomical Society, 418, 838

Wolfire M. G., Hollenbach D., Mckee C. F., 2010, The Astrophysical Journal, pp 1191-1207

Wong T., Blitz L., 2002, The Astrophysical Journal 569, 157
Xie L., et al., 2017, Monthly Notices of the Royal Astronomical Society, 000

Zasov A., Kasparova A., 2014, Astrophysics and Space Science. 353,595

This paper has been typeset from a TEX/LATEX file prepared by the author. 